\title{
Strong solutions for the Stochastic 3D LANS- $\alpha$ model driven by non-Gaussian Lévy noise
}

\author{
Gabriel Deugoué ${ }^{1}$ and Mamadou Sango
}

\begin{abstract}
We establish the existence, uniqueness and approximation of the strong solutions for the stochastic 3D LANS- $\alpha$ model driven by a non-Gaussian Lévy noise. Moreover, we also study the stability of solutions. In particular, we prove that under some conditions on the forcing terms, the strong solution converges exponentially in the mean square and almost surely exponentially to the stationary solution.
\end{abstract}

Key Words or Phrases: LANS- $\alpha$ model; Lévy noise; Strong solutions; Galerkin approximation; Exponential stability.

Mathematics Subject Classification (2010): 60H15 35R60 35R05.

\section{Introduction}

The purpose of this paper is the study of the stochastic $3 D$ Lagrangian averaged Navier-Stokes alpha (LANS- $\alpha$ ) model driven by a non-Gaussian Lévy noise. More precisely, let $D$ be a connected and bounded open subset of $\mathbb{R}^{3}$, with a $C^{2}$ boundary $\partial D$. Let $T>0$ a final time. We denote by $A$ the Stokes operator, and consider the system

$$
\left\{\begin{array}{l}
d(u-\alpha \Delta u)+\left[\nu(A u-\alpha \Delta(A u))+(u \cdot \nabla)(u-\alpha \Delta u)-\alpha(\nabla u)^{*} . \Delta u+\nabla p\right] d t \\
=F(t, u) d t+\int_{Z} \sigma(t, u(t-), z) \widetilde{N}(d t, d z), \text { in }(0, T) \times D \\
\nabla \cdot u=0, \text { in }(0, T) \times D \\
u=0, A u=0, \text { on }(0, T) \times \partial D \\
u(0)=u_{0}, \text { in } D
\end{array}\right.
$$

where $u=\left(u_{1}, u_{2}, u_{3}\right)$ and $p$ are unknown random fields on $[0, T] \times D$, representing respectively, the large scale (or averaged) velocity and the pressure in each point of $[0, T] \times D$, of an incompressible viscous fluid with constant density filling the domain $D$. The constants $\nu>0$ and $\alpha>0$ are given, and represent, respectively, the kinematic viscosity of the fluid, and the square of the spatial scale at which fluid motion is filtered. $u_{0}$ is a given initial velocity field, and the terms $F(t, u)$ and $\int_{Z} \sigma(t, u(t-), z) \widetilde{N}(d t, d z)$ represent random external forces depending eventually on $u$, where $\widetilde{N}$ is a compensated Poisson measure on a measure space $(Z, \mathcal{Z})$ endowed with a $\sigma$-finite measure $\mu$. Precise assumptions on the data of the problem are given below. The notation $v^{*}$ in the system (1) denotes the transpose of a vector $v$.

The deterministic counterpart of (1), also called viscous Camassa-Holm equations, or the Navier-Stokes alpha model, has been the object of intensive investigation both for its physical importance and as a nonlinear partial differential equation (see e.g. [24], [16], [29], [41] and the references therein). The initial motivation was to find a closure model for the 3D turbulence averaged Reynolds model; for more details, we refer to [24] and the references therein. A key interest in the model is the fact that it serves as a good approximation of the 3D Navier-Stokes equations. It is readily seen that when $\alpha=0$, the problem reduces to the usual 3D NavierStokes equations. Many important results have been obtained in the deterministic case. More precisely, the global well-posedness of weak solutions for the Lagrangian averaged Navier-Stokes alpha model on bounded domains has been established in [16] and [41] amongst others, and the global attractor was constructed in [16]. Similar results have been proved by Foias et al. in [24] in the case of periodic boundary conditions.

\footnotetext{
${ }^{1}$ Corresponding author, E-mail address:agdeugoue@yahoo.fr
} 
The study of the stochastic 3D Lagrangian averaged Navier-Stokes alpha model driven by Wiener noise was initiated by Caraballo et al. [11, 12, 13]. In [11, 12], they proved the existence and uniqueness of the stochastic $3 D$ LANS- $\alpha$ model under Lipschitz assumptions on the random forcing terms. The study of the asymptotic behavior of the probabilistic strong solution was investigated in [13]. We proved in [17] the existence of a probabilistic weak solution under continuity and linear growth assumptions on the forcing terms, thus extending the result in [11]. In the case of periodic boundary conditions, we studied in [18] the asymptotic behavior of the 3D stochastic LANS- $\alpha$ model when $\alpha$ approaches zero. We refer also to [19], [20] for related work.

In the last few years, there has been interest in the study of stochastic partial differential equations driven by jumps type noise. Much of motivation has come from mathematical physics and from financial mathematics. In many applications jumps processes provide more realistic models than continuous processes do.

In the presence case, the need for considering jump processes in the governing equations for turbulent motions of fluids is connected with the developments arising from the theory of turbulence of Kolmogorov ([33], [34]) and Obukhov [42]. Indeed their first theory of turbulence was challenged by Landau for not taking into account the effects of intermittency in the velocity fluctuations, on the scaling exponents that formed the backbone of the theory. In their revised version of the theory, Kolmogorov [35] and Obukhov [43] raised the prospect of dealing with the corrections in the scaling exponents by means of log-normal processes as far as the order two structure functions were concerned. In addressing the corrective measures related to intermittency Dubrulle [23], Leveque, She and Waymire ([48], [49]) discovered that log-Poisson processes were needed for higher-order structure functions. In order to account for this corrected influence of intermittency in the governing equations for the corresponding fluid, Navier Stokes equations driven by multiplicative jump processes had to be considered, since they are the ones that generate the discovered log-Poissonian effects. These Jumps processes, which are of Levy type in general, capture the large excursions and intermittency in the velocity field of the turbulent particles of the fluid. For a thorough discussion of this exciting topic, we refer to the recent works of Birnir ([3], [4]).

The existence and uniqueness of solutions for stochastic differential equations driven by jump noise has already been intensively investigated by many authors, see e.g. [31, 1, 2, 51, 32, 26, 27, $28,38,46,21,39,5,22,6,8,7,9]$ and the monograph by Peszat and Zabczyk [44]. There are few papers studying non-Lipschitz stochastic partial differential equations driven by Lévy type noise. The authors in [7] proved the existence and uniqueness of solutions to stochastic nonlinear beam equations driven by Lévy type noise. In [8], they also investigated the well-posedness for a class of stochastic equations of hydrodynamics driven by a non-Gaussian Lévy type noise. This class comprises the stochastic $2 D$ Navier-Stokes equations, the stochastic $2 D$ magnetohydrodynamic equations, the stochastic $2 D$ Boussineqs model for the Bénard convection, the stochastic $2 D$ magnetic Bénard problem, the stochastic $3 D$ Leray- $\alpha$ model for the Navier-Stokes equations and several stochastic shell models of turbulence. Brzeźniak et al.[9], established the existence and uniqueness of solutions for a general locally monotone operators and more general noise, thus extending the result in $[37,8]$. The method of the proof uses the Galerkin approximation and some local monotonicity arguments. However, our model does not fall in this framework. Indeed, this is due to the properties of the nonlinear term present in our model (see (24)). To the best of our knowledge, there is no result concerning the study of the stochastic $3 D$ LANS- $\alpha$ model driven by jump noise.

In this article, we prove the existence, uniqueness and approximation of the stochastic $3 D$ LANS- $\alpha$ model driven by discontinuous multiplicative noise (see Theorem 1 and Theorem 2). Compared to the work in [8], our method of the proof of Theorem 1 is different. We still use the Galerkin approximation combined with the techniques of stopping times and some convergence principles from functional analysis [52] as in $[11,10]$ but not, as opposed to [6] compactness argument. We shall use the result from [1] for the finite dimensional case to construct a sequence 
of solutions of approximated equations and obtain a priori estimates for those approximated solutions. Then we show that the limit of those approximated solutions solves the original equation by using the techniques of stopping times (see Lemma 2). To prove the existence of the pressure of problem (1), we apply the result in [36], see Remark 4.3. For this purpose, we need to estimate the fourth order moment of the stochastic integral with respect to a compensated Poisson measure. This is achieved by using the result in ([26], Corollary 3.1, Remark 3.6 or [40], Lemma 3.1) concerning the maximal inequality for stochastic integral with respect to a compensated Poisson measure. In Theorem 2, we prove that the whole sequence of the Galerkin approximation converges in mean square to the strong solution of the stochastic 3D LANS- $\alpha$ model. Moreover, we also investigate the stability of solutions. In particular, we prove that under some conditions on the forcing terms, the strong solution converges exponentially in the mean square and almost surely exponentially to the stationary solutions (see Theorem 3). The proof follows the approach in $[13,14,45]$. This article is therefore a generalization of the papers $[11,13]$ to the case of nonGaussian Lévy noise.

The paper is structured as follows. In Section 2, we will recall some preliminaries on stochastic integral with respect to a compensated Poisson measure. In Section 3, we recall some results concerning the nonlinear term in (1) and state the first main results. In Section 4, we rewrite our problem as an abstract evolution problem. In Section 5, we introduce the Galerkin approximation of our problem and derive a priori estimates for its solution. Then we prove the existence and approximation of strong solution for the stochastic 3D LANS- $\alpha$ model. In the last section, we analyze the stability of stationary solutions.

\section{Stochastic preliminaries}

Let $(\Omega, \mathcal{F}, \mathbb{P})$ be a complete probability space equipped with a filtration $\mathbb{F}=\left\{\mathcal{F}_{t}\right\}_{t \in[0, T]}$ satisfying the usual conditions. We denote by $\mathbb{E}$ the expectation with respect to $\mathbb{P}$. Let $(Z, \mathcal{Z}, \mu)$ be a measure space, where $\mu$ is a $\sigma$-finite measure. Let $p=(p(t), t \in D(p))$ be a stationary $\mathcal{F}_{t}$-Poisson point process on $Z$ with characteristic measure $\mu$, where $D(p)$ is a countable subset of $[0, \infty$ ) (see $[30,53]$ for the details on Poisson point processes). Denote by $N(d t, d z)$ the Poisson counting measure associated with $p$ and $\widetilde{N}(d t, d z):=N(d t, d z)-\mu(d z) d t$ be the compensated Poisson measure associated to $N(d t, d z)$.

Let $\mathcal{P}$ be a predictable $\sigma$-field, i.e., the $\sigma$-field generated by left continuous and $\mathbb{F}$-adapted real valued processes on $[0, T] \times \Omega$. We shall denote by $\mathcal{B F}$ the $\sigma$-field of the progressively measurable sets on $[0, T] \times \Omega$, i.e. $\mathcal{B \mathcal { F }}=\left\{A \subset[0, T] \times \Omega: \forall t \in[0, T], A \cap([0, t] \times \Omega) \in \mathcal{B}([0, t]) \otimes \mathcal{F}_{t}\right\}$. Consider a real separable Hilbert space $E$. Let $\mathcal{M}_{T}^{q}(\mathcal{P} \otimes \mathcal{Z}, d t \times \mathbb{P} \times \mu ; E), q \in[1, \infty)$ be the space of all (equivalence classes of) $\mathcal{P} \otimes \mathcal{Z}$-measurable functions $f:[0, T] \times \Omega \times Z \rightarrow E$ such that

$$
\mathbb{E} \int_{0}^{T} \int_{Z}\|f(s, ., z)\|_{E}^{q} \mu(d z) d s<\infty .
$$

Let $\mathcal{M}_{T}(\mathcal{P} \times \mathcal{Z}, N, E)$ be the space of all $\mathcal{P} \otimes \mathcal{Z}$-measurable functions $f:[0, T] \times \Omega \times Z \rightarrow E$ such that

$$
\mathbb{E} \int_{0}^{T} \int_{Z}\|f(s, ., z)\|_{E} N(d s, d z)<\infty .
$$

Here $\int_{0}^{T}\|f(s, ., z)\|_{E} N(d s, d z)(\omega)$ is understood to be the Lebesgue integral with respect to the measure $N(.,).(\omega)$ for every $\omega \in \Omega$ and is equal to the convergent sum (see [30]),

$$
\int_{0}^{T} \int_{Z}\|f(s, \omega, z)\|_{E} N(d s, d z)=\sum_{s \in(0, T] \cap D(p(\omega))} \| f\left(s, \omega, p(s, \omega) \|_{E} .\right.
$$

If $f:[0, T] \times \Omega \times Z \rightarrow E$ is a $\mathcal{B}([0, T]) \otimes \mathcal{F}_{T} \otimes \mathcal{Z}$-measurable function and $\mathbb{E} \int_{0}^{T} \int_{Z}\|f(s, ., z)\|_{E} N(d s, d z)<\infty$, then for every $\omega \in \Omega, f(., \omega,$.$) is \mathcal{B}([0, T]) \otimes \mathcal{Z}$-measurable 
and $\int_{0}^{T} \int_{Z}\|f(s, \omega, z)\|_{E} N(d s, d z)(\omega)<\infty, \mathbb{P}$-a.s., hence for almost all $\omega \in \Omega, f(., \omega,$.$) is Bochner$ integrable with respect to $N(d s, d z)(\omega)$ and we have for every $0 \leq t \leq T$

$$
\int_{0}^{t} \int_{Z} f(s, \omega, z) N(d s, d z)(\omega)=\sum_{s \in(0, t] \cap D(p(\omega))} f(s, \omega, p(s, \omega)), \mathbb{P}-a . s . .
$$

Denote by $\mathbb{D}([0, T] ; E)$ the space of all càdlàg paths from $[0, T]$ into $E$.

Now, we state some important properties of the stochastic integrals with respect to the compensated Poisson measure . Proofs of these properties and detailed discussions can be found in $[30]$ ( see also $[47,53])$.

Proposition 1. Let $f \in M_{T}^{2}(\mathcal{P} \otimes \mathcal{Z}, d t \times \mathbb{P} \times \mu ; E)$.

(i) The stochastic integral process $\int_{0}^{t} \int_{Z} f(s, ., z) \widetilde{N}(d s, d z), t \in[0, T]$ is a càdlàg 2-integrable martingale. More precisely, it has a modification which has càdlàg trajectories.

ii)

$$
\mathbb{E}\left\|\int_{0}^{t} \int_{Z} f(s, ., z) \tilde{N}(d s, d z)\right\|_{E}^{2}=\mathbb{E} \int_{0}^{t} \int_{Z}\|f(s, ., z)\|_{E}^{2} \mu(d z) d s, \text { for } t \in[0, T] .
$$

iii) If $f \in M_{T}^{2}(\mathcal{P} \otimes \mathcal{Z}, d t \times \mathbb{P} \times \mu ; E) \cap M_{T}^{1}(\mathcal{P} \otimes \mathcal{Z}, d t \times \mathbb{P} \times \mu ; E)$, then we have for each $t \in[0, T], \mathbb{P}$-a.s.

$$
\int_{0}^{t} \int_{Z} f(s, ., z) \tilde{N}(d s, d z)=\sum_{s \in(0, t] \cap D(p)} f(s, ., p(s))-\int_{0}^{t} \int_{Z} f(s, ., z) \mu(d z) d s .
$$

Remark 1. We may extend the stochastic integral to $\mathcal{P} \otimes \mathcal{Z}$-measurable functions $f$ satisfying

$$
\int_{0}^{T} \int_{Z}\|f(s, ., z)\|_{E}^{2} \mu(d z) d s<\infty ; \mathbb{P}-\text { a.s.. }
$$

In this case, the stochastic integral process $\int_{0}^{t} \int_{Z} f(s, ., z) \widetilde{N}(d s, d z), t \in[0, T]$ is a càdlàg 2integrable local martingale and for every stopping time $\tau \leq T$, we have

$$
\int_{0}^{t \wedge \tau} \int_{Z} f(s, ., z) \widetilde{N}(d s, d z)=\int_{0}^{t} \int_{Z} I_{[0, \tau]} f(s, ., z) \widetilde{N}(d s, d z) .
$$

\section{Some results about the nonlinear term and the first main re- sults}

\subsection{Notations and properties of the nonlinear term}

Following [11], we recall some properties regarding the nonlinear term $(u . \nabla)(u-\alpha \Delta u)-\alpha(\nabla u)^{*} . \Delta u$ appearing in (1).

We denote by $(.,$.$) and |$.$| , respectively, the scalar product and associated norm in \left(L^{2}(D)\right)^{3}$. We consider the scalar product in $\left(H_{0}^{1}(D)\right)^{3}$ defined by

$$
((u, v))=(u, v)+\alpha(\nabla u, \nabla v), \text { for } u, v \text { in }\left(H_{0}^{1}(D)\right)^{3},
$$

where its associated norm $\|$.$\| is, in fact, equivalent to the usual gradient norm. We denote by H$ the closure in $\left(L^{2}(D)\right)^{3}$ of the set $\mathcal{V}=\left\{v \in(\mathcal{D}(D))^{3}: \nabla v=0\right.$ in $\left.D\right\}$, and by $V$ the closure of $\mathcal{V}$ in $\left(H_{0}^{1}(D)\right)^{3}$. $H$ is a Hilbert space equipped with the inner product of $\left(L^{2}(D)\right)^{3}$, and $V$ is a Hilbert subspace of $\left(H_{0}^{1}(D)\right)^{3}$. Denote by $A$ the Stokes operator, with domain $D(A)=\left(H^{2}(D)\right)^{3} \cap V$, defined by

$$
A w=-\mathcal{P}(\Delta w), w \in D(A),
$$


where $\mathcal{P}$ is the projection operator from $\left(L^{2}(D)\right)^{3}$ onto $H$. Recall that $\partial D$ is $C^{2},|A w|$ defines in $D(A)$ a norm which is equivalent to the $\left(H^{2}(D)\right)^{3}$-norm, i.e. there exists a constant $c_{1}(D)>0$, depending only on $D$, such that

$$
\|w\|_{\left(H^{2}(D)\right)^{3}} \leq c_{1}(D)|A w|, \forall w \in D(A) .
$$

So, $D(A)$ is a Hilbert space with respect to the scalar product

$$
(u, w)_{D(A)}=(A v, A w) .
$$

For $u \in D(A)$ and $v \in\left(L^{2}(D)\right)^{3}$, we define $(u . \nabla) v$ as the element of $\left(H^{-1}(D)\right)^{3}$ given by

$$
\langle(u . \nabla) v, w\rangle=\sum_{i, j=1}^{3}\left\langle\partial_{i} v_{j}, u_{i} w_{j}\right\rangle
$$

for all $w \in\left(H_{0}^{1}(D)\right)^{3}$. Here $\langle.,$.$\rangle is the duality product between \left(H^{-1}(D)\right)^{3}$ and $\left(H_{0}^{1}(D)\right)^{3}$ (respectively between $H^{-1}(D)$ and $H_{0}^{1}(D)$.

There exists a constant $c_{2}(D)>0$ depending only on $D$, such that

$$
|\langle(u . \nabla) v, w\rangle| \leq c_{2}(D)|A u\|v \mid\| w \|,
$$

for all $(u, v, w) \in D(A) \times\left(L^{2}(D)\right)^{3} \times\left(H_{0}^{1}(D)\right)^{3}$.

Now, if $u \in D(A)$, then $(\nabla u)^{*} \in\left(H^{1}(D)\right)^{3 \times 3} \subset\left(L^{6}(D)\right)^{3 \times 3}$, and consequently for $v \in\left(L^{2}(D)\right)^{3}$, we have that $(\nabla u)^{*} \cdot v \in\left(L^{\frac{3}{2}}(D)\right)^{3} \subset\left(H^{-1}(D)\right)^{3}$, with

$$
\left\langle(\nabla u)^{*} . v, w\right\rangle=\sum_{i, j=1}^{3} \int_{D}\left(\partial_{j} u_{i}\right) v_{i} w_{j} d x, \text { for all } w \in\left(H_{0}^{1}(D)\right)^{3} .
$$

It follows that there exists a constant $c_{3}(D)>0$, depending only on $D$, such that

$$
\left|\left\langle(\nabla u)^{*} \cdot v, w\right\rangle\right| \leq c_{3}(D)\left|A u\|v \mid\| w \|, \forall(u, v, w) \in D(A) \times\left(L^{2}(D)\right)^{3} \times\left(H_{0}^{1}(D)\right)^{3} .\right.
$$

We have the following result (see [11])

Proposition 2. For all $(u, w) \in D(A) \times D(A)$, and all $v \in\left(L^{2}(D)\right)^{3}$, it follows that

$$
\langle(u . \nabla) v, w\rangle=-\left\langle(\nabla w)^{*} \cdot v, u\right\rangle .
$$

Consider now the bilinear form defined by

$$
b^{\#}(u, v, w)=\langle(u . \nabla) v, w\rangle+\left\langle(\nabla u)^{*} \cdot v, w\right\rangle,
$$

for $(u, v, w) \in D(A) \times\left(L^{2}(D)\right)^{3} \times\left(H_{0}^{1}(D)\right)^{3}$.

Proposition 3. The trilinear form $b^{\#}$ satisfies

$$
b^{\#}(u, v, w)=-b^{\#}(w, v, u), \forall(u, v, w) \in D(A) \times\left(L^{2}(D)\right)^{3} \times D(A) .
$$

and consequently

$$
b^{\#}(u, v, u)=0, \forall(u, v) \in D(A) \times\left(L^{2}(D)\right)^{3} .
$$

Moreover, there exists a constant $c(D)>0$, depending only on $D$ such that

$$
\begin{gathered}
\left|b^{\#}(u, v, w)\right| \leq c(D)|A u||v|\|w\|, \text { for all }(u, v, w) \in D(A) \times\left(L^{2}(D)\right)^{3} \times\left(H_{0}^{1}(D)\right)^{3}, \\
\left|b^{\#}(u, v, w)\right| \leq c(D)\|u\||v \| A u|, \text { for all }(u, v, w) \in D(A) \times\left(L^{2}(D)\right)^{3} \times D(A) .
\end{gathered}
$$

In particular, $b^{\#}$ is continuous on $D(A) \times\left(L^{2}(D)\right)^{3} \times\left(H_{0}^{1}(D)\right)^{3}$. 


\subsection{The first main results}

We assume that $F:[0, T] \times \Omega \times V \rightarrow\left(H^{-1}(D)\right)^{3}$ is a $\mathcal{B F} \otimes \mathcal{B}(V)$-measurable function and $\sigma:[0, T] \times \Omega \times V \times Z \rightarrow H$ is a $\mathcal{P} \otimes \mathcal{B}(V) \otimes \mathcal{Z}$-measurable function. Furthermore suppose that $F$ and $\sigma$ satisfy the following assumptions $\mathbb{P}$-a.s.:

$$
\begin{gathered}
\left\|F\left(t, v_{1}\right)-F\left(t, v_{2}\right)\right\|_{\left(H^{-1}(D)\right)^{3}}^{2} \leq L_{F}\left\|v_{1}-v_{2}\right\|^{2}, \\
\left\|F\left(t, v_{1}\right)\right\|_{\left(H^{-1}(D)\right)^{3}}^{2} \leq L_{0}\left(1+\left\|v_{1}\right\|^{2}\right), \\
\left|\sigma\left(t, v_{1}\right)\right|_{L^{2}(Z, \mu ; H)}^{2} \leq K_{0}\left(1+\left\|v_{1}\right\|^{2}\right), \\
\left|\sigma\left(t, v_{1}\right)-\sigma\left(t, v_{2}\right)\right|_{L^{2}(Z, \mu ; H)}^{2} \leq L_{\sigma}\left\|v_{1}-v_{2}\right\|^{2}, \\
\left|\sigma\left(t, v_{1}\right)\right|_{L^{4}(Z, \mu ; H)}^{4} \leq K\left(1+\left\|v_{1}\right\|^{4}\right),
\end{gathered}
$$

for all $v_{1}, v_{2} \in V$ and $\mathbb{P} \times d t$ a.e. in $\Omega \times[0, T]$, where $L_{F}, L_{0}, K_{0}, L_{\sigma}$ and $K$ are constants. Finally, we assume that $u_{0} \in L^{2}\left(\Omega, \mathcal{F}_{0}, \mathbb{P} ; V\right)$.

Now, we are going to define a solution of our problem (1)

Definition 1. An V-valued càdlàg $\mathbb{F}$-adapted process $u$ is called a variational solution of problem (1) if

1) $u \in L^{2}\left(\Omega ; L^{2}(0, T ; D(A))\right) \cap L^{2}\left(\Omega ; L^{\infty}(0, T ; V)\right)$,

2) the following equality holds $\mathbb{P}$-a.s.:

$$
\begin{aligned}
& ((u(t), w))+\nu \int_{0}^{t}(u(s)+\alpha A u(s), A w) d s+\int_{0}^{t} b^{\#}(u(s), u(s)-\alpha \Delta u(s), w) d s \\
& =\left(\left(u_{0}, w\right)\right)+\int_{0}^{t}\langle F(s, u(s)), w\rangle d s+\int_{0}^{t} \int_{Z}(\sigma(s, u(s-), z), w) \widetilde{N}(d s, d z),
\end{aligned}
$$

for all $w \in D(A), t \in[0, T]$.

Remark 2. Equation (21) is derived from (1) by multiplying the first equation in (1) by $w \in D(A)$, taking into account the definition of the scalar product $((.,)$.$) , the expression of b^{\#}$ and the equality (11).

Our first main results are the following.

Theorem 1. 1) Assume that conditions (16)-(20) are satisfied and $u_{0} \in L^{4}\left(\Omega, \mathcal{F}_{0}, \mathbb{P} ; V\right)$. Then there exists a unique variational solution $u$ to problem (1), which satisfies $u \in L^{4}(\Omega ; \mathbb{D}([0, T] ; V)) \cap$ $L^{4}\left(\Omega ; L^{2}(0, T ; D(A))\right.$. In fact, there exists a constant $C>0$, depending only on $\alpha, \nu, T, L_{F}, L_{0}, K_{0}$ and $K$ such that

$$
\mathbb{E}\left[\sup _{0 \leq t \leq T}\|u(t)\|^{4}+\int_{0}^{T}\|u(s)\|^{2}\|u(s)\|_{D(A)}^{2} d s+\left(\int_{0}^{T}\|u(s)\|_{D(A)}^{2} d s\right)^{2}\right] \leq C\left(\mathbb{E}\left(\left\|u_{0}\right\|^{4}\right)+1\right) .
$$

2) Moreover, associated to the variational solution $u$, there exists a unique $p \in L^{2}\left(\Omega, \mathcal{F}_{t}, \mathbb{P} ; H^{-1}\left(0, t ; H^{-1}(D)\right)\right)$, for all $t \in[0, T]$, such that $\mathbb{P}$-a.s.

$$
\begin{aligned}
& d(u-\alpha \Delta u)+\left[\nu(A u-\alpha \Delta(A u))+(u . \nabla)(u-\alpha \Delta u)-\alpha(\nabla u)^{*} \cdot \Delta u+\nabla p\right] d t \\
& =F(t, u) d t+\int_{Z} \sigma(t, u(t-), z) \widetilde{N}(d t, d z), \text { in }\left(\mathcal{D}^{\prime}\left((0, T) \times D ; D(A)^{\prime}\right)\right)^{3}, \\
& \int_{D} p d x=0 \text { in } \mathcal{D}^{\prime}(0, T) .
\end{aligned}
$$


Theorem 2. Suppose that the hypotheses of Theorem 1 hold. Let $\left(u_{n}\right)_{n \in \mathbb{N}}$ the unique solution of the Galerkin approximation of problem (1) (see (28) below) and u the unique solution of problem (1). We have the following convergence results:

$$
\begin{aligned}
& \lim _{n \rightarrow \infty} \mathbb{E}\left\|u(t)-u_{n}(t)\right\|^{2}=0, \\
& \lim _{n \rightarrow \infty} \mathbb{E} \int_{0}^{t}\left\|u(s)-u_{n}(s)\right\|_{D(A)}^{2} d s=0,
\end{aligned}
$$

for all $t \in[0, T]$.

Remark 3. (1)- Condition (20) is needed to show uniform estimates of the fourth moment of the approximating solution, see Proposition 4. Conditions (16)-(19) are sufficient to prove the existence and uniqueness of the solution.

(2)- Note that if a process $u(t), t \in[0, T]$ is adapted and càdlàg, then the left-limit process $u(t-)$ is left continuous and adapted. Hence the process $u(t-), t \in[0, T]$ is predictable. In such case, Definition 1 is reasonable since the integrand in the stochastic integral is predictable.

\section{Abstract formulation of the problem}

Following [11], we are going to rewrite our model as an abstract problem.

We identify $V$ with its topological dual $V^{\prime}$ and we consider $D(A)$ as a subspace of $V^{\prime}$. We then have the Gelfand triple $D(A) \subset V=V^{\prime} \subset D(A)^{\prime}$.

Recall that the scalar product in $V$ is $((u, v))=(u, v)+\alpha(\nabla u, \nabla v)$ and its associated norm is denoted by $\|$.$\| .$

The scalar product on $D(A)$ is $(u, v)_{D(A)}=(A u, A v)$.

We denote by $\langle.,$.$\rangle the duality product between D(A)^{\prime}$ and $D(A)$. Define the operator $\widetilde{A}$ by

$$
\langle\widetilde{A} u, v\rangle=\nu(A u, v)+\nu \alpha(A u, A v)
$$

for all $u, v \in D(A)$. It is clear that for all $v \in D(A)$,

$$
2\langle\widetilde{A} v, v\rangle \geq 2 \nu \alpha|A v|^{2},
$$

and, if we denote by $\mu_{k}$ and $w_{k}, k \geq 1$, the eigenvalues and their corresponding eigenvectors associated to $A$, then

$$
\left\langle\widetilde{A} w_{k}, v\right\rangle=\nu \mu_{k}\left(\left(w_{k}, v\right)\right)
$$

Taking $\widetilde{\alpha}=2 \nu \alpha$, we have (see the details of the proof in [13])

(a) $\widetilde{A}$ is a linear continuous operator $\widetilde{A} \in \mathcal{L}\left(D(A), D(A)^{\prime}\right)$ such that

(a1) $\widetilde{A}$ is self-adjoint,

(a2) there is a constant $\widetilde{\alpha}>0$, such that $2\langle\widetilde{A} v, v\rangle \geq \widetilde{\alpha}\|v\|_{D(A)}^{2}$, for all $v \in D(A)$.

There exists a orthonormal basis $\left\{v_{k}, k \geq 1\right\} \subset D(A)$ of $V$ and an increasing sequence $\left\{\lambda_{k} ; k \geq 1\right\} \subset(0, \infty)$ such that

$$
\widetilde{A} v_{k}=\lambda_{k} v_{k}
$$

where $\lambda_{k}=\nu \mu_{k}$ and $v_{k}=\frac{w_{k}}{\sqrt{1+\alpha \mu_{k}}}$.

On the other hand, denote

$$
\begin{gathered}
\langle\widetilde{B}(u, v), w\rangle=b^{\#}(u, v-\alpha \Delta v, w), \text { for }(u, v, w) \text { in }(D(A))^{3} \\
((\widetilde{F}(t, u), w))=\langle F(t, u), w\rangle, \text { for }(u, w) \in V \times D(A) .
\end{gathered}
$$


Then it is straightforward to check that if we take

$$
c_{1}=(1+\alpha) c_{1}(D) c(D) \text { and } L_{\widetilde{F}}=L_{F},
$$

then we obtain

(b) $\widetilde{B}: D(A) \times D(A) \rightarrow D(A)^{\prime}$ is a bilinear mapping such that

(b1) $\langle\widetilde{B}(u, v), u\rangle=0$, for all $u, v \in D(A)$,

(b2) $\|\widetilde{B}(u, v)\|_{D(A)^{\prime}} \leq c_{1}\|u\|\|v\|_{D(A)}$, for all $(u, v) \in D(A) \times D(A)$,

(b3) $|\langle\widetilde{B}(u, v), w\rangle| \leq c_{1}\|u\|_{D(A)}\|v\|_{D(A)}\|w\|$, for all $u, v, w \in D(A)$.

(c) $\widetilde{F}:[0, T] \times \Omega \times V \rightarrow V$ is a random mapping such that

(c1) $\widetilde{F}$ is $\mathcal{B F} \otimes \mathcal{B}(V)$ - measurable,

$(c 2)\left\|\widetilde{F}\left(t, v_{1}\right)-\widetilde{F}\left(t, v_{2}\right)\right\|^{2} \leq L_{\widetilde{F}}\left\|v_{1}-v_{2}\right\|^{2}$,

(c3) $\left\|\widetilde{F}\left(t, v_{1}\right)\right\|^{2} \leq L_{0}\left(1+\left\|v_{1}\right\|^{2}\right)$, for $\mathbb{P} \times d t$ a.e. for all $v_{1}, v_{2} \in V$.

Let $I$ denote the identity operator in $H$, and define $\widetilde{\sigma}(t, u, z)$ as

$$
\widetilde{\sigma}(t, u, z)=(I+\alpha A)^{-1} \circ \mathcal{P} \circ \sigma(t, u, z),
$$

for $u \in V, z \in Z$.

We have

$$
\left\|(I+\alpha A)^{-1} f\right\|^{2} \leq \frac{1}{1+\alpha \mu_{1}}|f|^{2}
$$

for all $f \in H$. Also observe that

$$
\|u\| \leq c_{2}\|u\|_{D(A)}, \forall u \in D(A)
$$

where $c_{2}=\frac{\sqrt{1+\alpha \mu_{1}}}{\mu_{1}}$. See the proof in [13].

Consequently, taking

$$
L_{\widetilde{\sigma}}=\frac{L_{\sigma}}{1+\alpha \mu_{1}},
$$

we obtain

(d) $\widetilde{\sigma}:[0, T] \times \Omega \times V \times Z \rightarrow V$ is a random mapping such that

$$
\begin{aligned}
& (d 1) \tilde{\sigma} \text { is a } \mathcal{P} \otimes \mathcal{B}(V) \otimes \mathcal{Z}-\text { measurable function, } \\
& (d 2)\left\|\widetilde{\sigma}\left(t, v_{1}\right)-\widetilde{\sigma}\left(t, v_{2}\right)\right\|_{L^{2}(Z, \mu ; V)}^{2} \leq L_{\widetilde{\sigma}}\left\|v_{1}-v_{2}\right\|^{2}, \\
& (d 3)\left\|\widetilde{\sigma}\left(t, v_{1}\right)\right\|_{L^{2}(Z, \mu ; V)}^{2} \leq \frac{K_{0}}{1+\alpha \mu_{1}}\left(1+\left\|v_{1}\right\|^{2}\right), \\
& (d 4)\left\|\widetilde{\sigma}\left(t, v_{1}\right)\right\|_{L^{4}(Z, \mu ; V)}^{4} \leq \frac{K}{\left(1+\alpha \mu_{1}\right)^{2}}\left(1+\left\|v_{1}\right\|^{4}\right) .
\end{aligned}
$$

In the sequel, we denote $K_{0}^{\prime}=\frac{K_{0}}{1+\alpha \mu_{1}}$ and $K^{\prime}=\frac{K}{\left(1+\alpha \mu_{1}\right)^{2}}$ for simplicity. Next, for $(t, u, w, z) \in[0, T] \times V \times D(A) \times Z$,

$$
\begin{aligned}
(\sigma(t, u, z), w) & =((I+\alpha A) \widetilde{\sigma}(t, u, z), w) \\
& =((\widetilde{\sigma}(t, u, z), w))
\end{aligned}
$$


and for all $u \in L^{2}(\Omega ; \mathbb{D}([0, T] ; V)),(t, w) \in[0, T] \times D(A)$, it follows that

$$
\begin{aligned}
& \int_{0}^{t} \int_{Z}(\sigma(s, u(s-), z), w) \tilde{N}(d s, d z), \\
& =\int_{0}^{t} \int_{Z}((\widetilde{\sigma}(s, u(s-), z), w)) \tilde{N}(d s, d z) .
\end{aligned}
$$

Consequently, in this abstract framework, a variational solution of problem (1) is equivalently a stochastic process $u$ which is càdlàg and adapted to the filtration $\mathbb{F}$ such that

$$
u \in L^{2}\left(\Omega ; L^{2}(0, T ; D(A))\right) \cap L^{2}(\Omega ; \mathbb{D}([0, T] ; V)),
$$

and such that the equation

$$
\begin{aligned}
& u(t)+\int_{0}^{t} \widetilde{A} u(s) d s+\int_{0}^{t} \widetilde{B}(u(s), u(s)) d s \\
& =u_{0}+\int_{0}^{t} \widetilde{F}(s, u(s)) d s+\int_{0}^{t} \int_{Z} \widetilde{\sigma}(s, u(s-), z) \widetilde{N}(d s, d z),
\end{aligned}
$$

is satisfied in $D(A)^{\prime}, \mathbb{P}$-a.s., for all $t \in[0, T]$.

We then state an equivalent formulation of problem (1).

Definition 2. An $V$-valued càdlàg $\mathbb{F}$-adapted process $u$ is called a variational solution of problem (1) if

(1) $u \in L^{2}\left(\Omega ; L^{2}(0, T ; D(A))\right) \cap L^{2}(\Omega ; \mathbb{D}([0, T] ; V))$,

(2) the following equality holds in $D(A)^{\prime}, \mathbb{P}$-a.s.

$$
\begin{aligned}
& u(t)+\int_{0}^{t} \widetilde{A} u(s) d s+\int_{0}^{t} \widetilde{B}(u(s), u(s)) d s \\
& =u_{0}+\int_{0}^{t} \widetilde{F}(s, u(s)) d s+\int_{0}^{t} \int_{Z} \widetilde{\sigma}(s, u(s-), z) \widetilde{N}(d s, d z),
\end{aligned}
$$

for all $t \in[0, T]$.

\section{Proof of Theorem 1 and Theorem 2}

Before starting the proof of our main results, we state the following version of the Gronwall Lemma whose proof can be found in [15].

Lemma 1. Let $X, Y, I$ and $\Phi$ be non-negative processes and $Z$ be a non-negative integrable random variable. Assume that $I$ is non-decreasing and that there exist non-negative constants $C, \alpha, \beta, \gamma, \delta$ and $T$ satisfying firstly

$$
\begin{aligned}
& \int_{0}^{T} \Phi(s) d s \leq C, \text { a.s. } \\
& 2 \beta e^{C} \leq 1, \quad 2 \delta e^{C} \leq \alpha
\end{aligned}
$$

and secondly for all $t \in[0, T]$, there exists a constant $\widetilde{C}>0$, such that

$$
\begin{aligned}
& X(t)+\alpha Y(t) \leq Z+\int_{0}^{t} \Phi(r) X(r) d r+I(t), a . s . \\
& \mathbb{E} I(t) \leq \beta \mathbb{E} X(t)+\gamma \int_{0}^{t} \mathbb{E} X(s) d s+\delta \mathbb{E} Y(t)+\widetilde{C} .
\end{aligned}
$$

If $X \in L^{\infty}([0, T] \times \Omega)$, then we have

$$
\mathbb{E}[X(t)+\alpha Y(t)] \leq 2 \exp \left(C+2 t \gamma e^{C}\right)(\mathbb{E} Z+\widetilde{C}), t \in[0, T] .
$$




\subsection{Proof of Theorem 1}

The proof of Theorem 1 uses the Galerkin approximation. Using the results from [1], we prove the existence, uniqueness and certain uniform estimates for the sequence $\left(u_{n}\right)_{n}$ of the approximation. Then, as in $[10,11]$, we use the properties of stopping times and some basic convergence principles from functional analysis to prove the existence of the solution. We will split the proof into seven steps.

\section{Step1: Construction of an approximating sequence}

We take the orthonormal basis $\left\{v_{k} ; k \geq 1\right\} \subset D(A)$ of $\mathrm{V}$, satisfying $\widetilde{A} v_{k}=\lambda_{k} v_{k}$, see (23). For each integer $n \geq 1$, we denote by $H_{n}$ the vector space spanned by $\left\{v_{1}, \ldots, v_{n}\right\}$ and $\Pi_{n}$ the projection of $D(A)^{\prime}$ onto $H_{n}$, that is

$$
\Pi_{n} x=\sum_{k=1}^{n}\left\langle x, v_{k}\right\rangle v_{k}, \text { for } x \in D(A)^{\prime} .
$$

The restriction of $\Pi_{n}$ on $V$ is the orthogonal projection of $V$ onto $H_{n}$.

We consider the finite dimensional problem on $H_{n}$ given by

$$
\left\{\begin{array}{l}
d u_{n}(t)=\Pi_{n} G\left(t, u_{n}(t)\right) d t+\int_{Z} \Pi_{n} \widetilde{\sigma}\left(t, u_{n}(t-), z\right) \tilde{N}(d t, d z), \\
u_{n}(0)=\Pi_{n} u_{0}
\end{array}\right.
$$

where $G(t, u)=-\widetilde{A} u-\widetilde{B}(u, u)+\widetilde{F}(t, u)$, for $u \in D(A)$, and $t \leq T$.

Using the result in [1], Theorem 3.1, the system (28) has a unique adapted $H_{n}$-valued càdlàg local strong solution $u_{n}$ since $G$ is locally Lipschitz and $\widetilde{\sigma}_{n}:=\Pi_{n} \widetilde{\sigma}$ is globally Lipschitz. The estimates below extend the solution to any time interval $[0, T]$.

Step 2: A priori estimates for the approximating sequence

The proposition below gives some estimates of the sequence $\left(u_{n}\right)_{n \in \mathbb{N}}$.

Proposition 4. Let the assumptions be as in Theorem 1. Then there exists positive constants $C$ and $\widetilde{C}$ such that,

$$
\begin{aligned}
& \text { 1) } \sup _{n} \mathbb{E}\left(\sup _{s \in[0, T]}\left\|u_{n}(s)\right\|^{2}+2 \nu \alpha \int_{0}^{T}\left\|u_{n}(s)\right\|_{D(A)}^{2} d s\right) \leq C\left(\mathbb{E}\left(\left\|u_{0}\right\|^{2}\right)+1\right) \\
& \text { 2) } \sup _{n} \mathbb{E}\left[\sup _{s \in[0, T]}\left\|u_{n}(s)\right\|^{4}+\int_{0}^{T}\left\|u_{n}(s)\right\|^{2}\left\|u_{n}(s)\right\|_{D(A)}^{2} d s\right] \leq \widetilde{C}\left(\mathbb{E}\left(\left\|u_{0}\right\|^{4}\right)+1\right), \\
& \text { 3) } \sup _{n} \mathbb{E}\left(\int_{0}^{T}\left\|u_{n}(s)\right\|_{D(A)}^{2} d s\right)^{2} \leq \widetilde{C}\left(\mathbb{E}\left(\left\|u_{0}\right\|^{4}\right)+1\right) .
\end{aligned}
$$

Proof. By Itô's formula ([30]) and property $(b 1)$ of $\widetilde{B}$, we obtain for all $t \in[0, T]$,

$$
\begin{aligned}
& \left\|u_{n}(t)\right\|^{2}+2 \int_{0}^{t}\left\langle\widetilde{A} u_{n}(s), u_{n}(s)\right\rangle d s \\
& =2 \int_{0}^{t}\left(\left(u_{n}(s), \Pi_{n} \widetilde{F}\left(s, u_{n}(s)\right)\right) d s+\left\|\Pi_{n} u_{0}\right\|^{2}+2 \int_{0}^{t} \int_{Z}\left(\left(u_{n}(s-), \widetilde{\sigma}_{n}\left(s, u_{n}(s-), z\right)\right)\right) \widetilde{N}(d s, d z)\right. \\
& +\int_{0}^{t} \int_{Z}\left(\left\|u_{n}(s-)+\widetilde{\sigma}_{n}\left(s, u_{n}(s-), z\right)\right\|^{2}-\left\|u_{n}(s-)\right\|^{2}-2\left(\left(u_{n}(s-), \widetilde{\sigma}_{n}\left(s, u_{n}(s-), z\right)\right)\right)\right) N(d s, d z) .
\end{aligned}
$$

Using relation (a2), condition $(c 3)$ and the fact that $\|x\|^{2}-\|y\|^{2}+\|x-y\|^{2}=2((x-y, x))$ for 
$x, y \in V$, we rewrite (32) as follows

$$
\begin{aligned}
& \left\|u_{n}(t)\right\|^{2}+\widetilde{\alpha} \int_{0}^{t}\left\|u_{n}(s)\right\|_{D(A)}^{2} d s \\
& \leq\left\|\Pi_{n} u_{0}\right\|^{2}+C T+C \int_{0}^{t}\left\|u_{n}(s)\right\|^{2} d s+2 \int_{0}^{t} \int_{Z}\left(\left(u_{n}(s-), \widetilde{\sigma}_{n}\left(s, u_{n}(s-), z\right)\right)\right) \widetilde{N}(d s, d z) \\
& +\int_{0}^{t} \int_{Z}\left\|\widetilde{\sigma}_{n}\left(s, u_{n}(s-), z\right)\right\|^{2} N(d s, d z) .
\end{aligned}
$$

We proceed as done in [15] and use Lemma 1. Therefore, we set for $t \in[0, T]$

$$
\begin{aligned}
X(t) & :=\sup _{0 \leq s \leq t}\left\|u_{n}(s)\right\|^{2}, \\
Y(t) & :=\widetilde{\alpha} \int_{0}^{t}\left\|u_{n}(s)\right\|_{D(A)}^{2} d s, \\
I(t) & :=\sup _{0 \leq s \leq t}\left(2 \int_{0}^{s} \int_{Z}\left(\left(u_{n}(r-), \widetilde{\sigma}_{n}\left(r, u_{n}(r-), z\right)\right)\right) \widetilde{N}(d r, d z)+\int_{0}^{s} \int_{Z}\left\|\widetilde{\sigma}_{n}\left(r, u_{n}(r-), z\right)\right\|^{2} N(d r, d z)\right) .
\end{aligned}
$$

We will handle the two summands in $I(t)$ separately. Let us denote

$$
V_{n}(t)=\int_{0}^{t} \int_{Z}\left(\left(u_{n}(r-), \widetilde{\sigma}_{n}\left(r, u_{n}(r-), z\right)\right)\right) \tilde{N}(d r, d z) .
$$

Since $V_{n}(t)$ is a local martingale, so one can always choose an increasing sequence of stopping times $\left\{\tau_{n}^{N}\right\}_{N}$ such that $\tau_{n}^{N} \rightarrow \infty$ as $N \rightarrow \infty$ and $V_{n}(t \wedge$.$) is a martingale. Hence, on the$ basis of Fatou's Lemma, it's enough to assume that $V_{n}(t)$ is a martingale. By applying first the Burkholder-Davis-Gundy's inequality (see [30]), condition (d3), then the Hölder's inequality, and finally the Young's inequality, we get

$$
\begin{aligned}
& \mathbb{E} \sup _{0 \leq s \leq t} \int_{0}^{s} \int_{Z}\left(\left(u_{n}(r-), \widetilde{\sigma}_{n}\left(r, u_{n}(r-), z\right)\right)\right) \widetilde{N}(d r, d z) \\
& \leq C \mathbb{E}\left(\int_{0}^{t} \int_{Z}\left\|u_{n}(s)\right\|^{2}\left\|\widetilde{\sigma}_{n}\left(s, u_{n}(s), z\right)\right\|^{2} \mu(d z) d s\right)^{\frac{1}{2}} \\
& \leq C\left(\epsilon \mathbb{E} \sup _{s \in[0, t]}\left\|u_{n}(s)\right\|^{2}\right)^{\frac{1}{2}}\left[\frac{1}{\epsilon} \mathbb{E} \int_{0}^{t} K_{0}^{\prime}\left(1+\left\|u_{n}(s)\right\|^{2}\right) d s\right]^{\frac{1}{2}} \\
& \leq C \epsilon \mathbb{E} \sup _{s \in[0, t]}\left\|u_{n}(s)\right\|^{2}+\frac{C}{\epsilon} \mathbb{E} \int_{0}^{t} K_{0}^{\prime}\left(1+\left\|u_{n}(s)\right\|^{2}\right) d s \\
& \leq \frac{C K_{0}^{\prime} t}{\epsilon}+C \epsilon \mathbb{E} X(t)+\frac{C K_{0}^{\prime}}{\epsilon} \mathbb{E} \int_{0}^{t} X(s) d s,
\end{aligned}
$$

where $\epsilon>0$.

Next, we will deal with the second term in $I(t)$. Taking into account that the process

$$
\int_{0}^{t} \int_{Z}\left\|\widetilde{\sigma}_{n}\left(r, u_{n}(r-), z\right)\right\|^{2} N(d r, d z)
$$


has only positive jumps, we obtain

$$
\begin{aligned}
& \mathbb{E} \sup _{0 \leq s \leq t} \int_{0}^{s} \int_{Z}\left\|\widetilde{\sigma}_{n}\left(r, u_{n}(r-), z\right)\right\|^{2} N(d r, d z) \\
& \leq \mathbb{E} \int_{0}^{t} \int_{Z}\left\|\widetilde{\sigma}_{n}\left(r, u_{n}(r-), z\right)\right\|^{2} N(d r, d z) \\
& =\mathbb{E} \int_{0}^{t} \int_{Z}\left\|\widetilde{\sigma}_{n}\left(r, u_{n}(r-), z\right)\right\|^{2} \mu(d z) d r \\
& \leq \mathbb{E} \int_{0}^{t} K_{0}^{\prime}\left(1+\left\|u_{n}(r)\right\|^{2}\right) d r \\
& \leq K_{0}^{\prime} t+K_{0}^{\prime} \int_{0}^{t} \mathbb{E} X(s) d s,
\end{aligned}
$$

where we also used condition (d3). By combining the last two inequalities, it follows that for $t \in[0, T]$,

$$
\mathbb{E} I(t) \leq\left(\frac{C K_{0}^{\prime}}{\epsilon}+K_{0}^{\prime}\right) t+C \epsilon \mathbb{E} X(t)+\left(\frac{C K_{0}^{\prime}}{\epsilon}+K_{0}^{\prime}\right) \mathbb{E} \int_{0}^{t} X(s) d s .
$$

Thus the processes $X, Y$ and $I$ satisfy the conditions of Lemma 1 . Hence there exists a constant $\widetilde{C}>0$ such that

$$
\mathbb{E}(X(t)+\widetilde{\alpha} Y(t)] \leq\left(\mathbb{E}\left(\left\|u_{0}\right\|^{2}\right)+1\right), t \in[0, T]
$$

that is

$$
\mathbb{E}\left[\sup _{s \in[0, t]}\left\|u_{n}(s)\right\|^{2}+\widetilde{\alpha} \int_{0}^{t}\left\|u_{n}(s)\right\|_{D(A)}^{2} d s\right] \leq \widetilde{C}\left(\mathbb{E}\left(\left\|u_{0}\right\|^{2}\right)+1\right), t \in[0, T],
$$

which completes the proof of 1 ) .

By Applying the finite dimensional Itô's formula (see [30]) to the function $\|\cdot\|_{V}^{2 p}$, for $p=2$ and the process $u_{n}$, we obtain

$$
\begin{aligned}
& \left\|u_{n}(t)\right\|^{2 p}=\left\|\Pi_{n} u_{0}\right\|^{2 p}+2 p \int_{0}^{t}\left\|u_{n}(s)\right\|^{2(p-1)}\left\langle\Pi_{n} G\left(s, u_{n}(s)\right), u_{n}(s)\right\rangle d s \\
& +2 p \int_{0}^{t} \int_{Z}\left\|u_{n}(s-)\right\|^{2(p-1)}\left(\left(u_{n}(s-), \widetilde{\sigma}_{n}\left(s, u_{n}(s-), z\right)\right)\right) \widetilde{N}(d s, d z) \\
& +\int_{0}^{t} \int_{Z}\left\|u_{n}(s-)+\widetilde{\sigma}_{n}\left(s, u_{n}(s-), z\right)\right\|^{2 p}-\left\|u_{n}(s-)\right\|^{2 p} \\
& -2 p\left\|u_{n}(s-)\right\|^{2(p-1)}\left(\left(u_{n}(s-), \widetilde{\sigma}_{n}\left(s, u_{n}(s-), z\right)\right)\right) N(d s, d z) .
\end{aligned}
$$

Again using the properties (b1) of $\widetilde{B}$, the coercivity (a2) of $\widetilde{A}$ and condition $(c 3)$, we obtain

$$
\begin{aligned}
& \left\|u_{n}(t)\right\|^{2 p}+p \widetilde{\alpha} \int_{0}^{t}\left\|u_{n}(r)\right\|^{2(p-1)}\left\|u_{n}(r)\right\|_{D(A)}^{2} d r \leq\left\|\Pi_{n} u_{0}\right\|^{2 p}+p \int_{0}^{t} C\left(1+\left\|u_{n}(s)\right\|^{2 p}\right) d s+ \\
& 2 p \int_{0}^{t} \int_{Z}\left\|u_{n}(s-)+\widetilde{\sigma}_{n}\left(s, u_{n}(s-), z\right)\right\|^{2 p}-\left\|u_{n}(s-)\right\|^{2 p} \\
& \quad-2 p\left\|u_{n}(s-)\right\|^{2(p-1)}\left(\left(u_{n}(s-), \widetilde{\sigma}_{n}\left(s, u_{n}(s-), z\right)\right)\right) N(d s, d z) \\
& +2 p \int_{0}^{t} \int_{Z}\left\|u_{n}(s-)\right\|^{2(p-1)}\left(\left(u_{n}(s-), \widetilde{\sigma}_{n}\left(s, u_{n}(s-), z\right)\right)\right) \widetilde{N}(d s, d z) .
\end{aligned}
$$


Taking the supremum over $[0, t]$ on both sides of the above equality yields

$$
\begin{aligned}
& \sup _{s \in[0, t]}\left\|u_{n}(s)\right\|^{2 p}+p \widetilde{\alpha} \sup _{s \in[0, t]} \int_{0}^{s}\left\|u_{n}(r)\right\|^{2(p-1)}\left\|u_{n}(r)\right\|_{D(A)}^{2} d r \leq\left\|\Pi_{n} u_{0}\right\|^{2 p}+p C \int_{0}^{t}\left(1+\left\|u_{n}(s)\right\|^{2 p}\right) d s \\
& +2 p \sup _{s \in[0, t]} \int_{0}^{s} \int_{Z}\left\|u_{n}(r-)\right\|^{2(p-1)}\left(\left(u_{n}(r-), \widetilde{\sigma}_{n}\left(r, u_{n}(r-), z\right)\right)\right) \widetilde{N}(d r, d z) \\
& +\sup _{s \in[0, t]} \int_{0}^{s} \int_{Z}\left\|u_{n}(r-)+\widetilde{\sigma}_{n}\left(r, u_{n}(r-), z\right)\right\|^{2 p}-\left\|u_{n}(r-)\right\|^{2 p} \\
& -2 p\left\|u_{n}(r-)\right\|^{2(p-1)}\left(\left(u_{n}(r-), \widetilde{\sigma}_{n}\left(r, u_{n}(r-), z\right)\right)\right) N(d r, d z) \\
& \leq\left\|\Pi_{n} u_{0}\right\|^{2 p}+p C t+p C \int_{0}^{t}\left\|u_{n}(s)\right\|^{2 p} d s+I_{1}(t)+I_{2}(t),
\end{aligned}
$$

where

$$
\begin{aligned}
& I_{1}(t)=2 p \sup _{s \in[0, t]} \int_{0}^{s} \int_{Z}\left\|u_{n}(r-)\right\|^{2(p-1)}\left(\left(u_{n}(r-), \widetilde{\sigma}_{n}\left(r, u_{n}(r-), z\right)\right)\right) \widetilde{N}(d r, d z), \\
& I_{2}(t)=\sup _{s \in[0, t]} \int_{0}^{s} \int_{Z}\left\|u_{n}(r-)+\widetilde{\sigma}_{n}\left(r, u_{n}(r-), z\right)\right\|^{2 p} \\
& -\left\|u_{n}(r-)\right\|^{2 p}-2 p\left\|u_{n}(r-)\right\|^{2(p-1)}\left(\left(u_{n}(r-), \widetilde{\sigma}_{n}\left(r, u_{n}(r-), z\right)\right)\right) N(d r, d z) .
\end{aligned}
$$

Applying the Burkholder-Davis-Gundy's inequality, condition ( $d 3)$, Young's inequality and the inequality $|x|^{2 p-2} \leq 1+|x|^{2 p}$, for $p \geq 2$, we obtain

$$
\begin{aligned}
\mathbb{E} I_{1}(t) & \leq 2 p C \mathbb{E}\left(\int_{0}^{t} \int_{Z}\left\|u_{n}(s)\right\|^{4(p-1)}\left\|u_{n}(s)\right\|^{2}\left\|\widetilde{\sigma}_{n}\left(s, u_{n}(s-), z\right)\right\|^{2} \mu(d z) d s\right)^{\frac{1}{2}} \\
& \leq 2 p C \mathbb{E}\left(\int_{0}^{t} \int_{Z}\left\|u_{n}(s)\right\|^{4 p-2}\left\|\widetilde{\sigma}_{n}\left(s, u_{n}(s-), z\right)\right\|^{2} \mu(d z) d s\right)^{\frac{1}{2}} \\
& \leq 2 p C \mathbb{E}\left(\int_{0}^{t}\left\|u_{n}(s)\right\|^{4 p-2}\left(K_{0}^{\prime}\left(1+\left\|u_{n}(s)\right\|^{2}\right)\right) d s\right)^{\frac{1}{2}} \\
& =2 p C\left(\epsilon \mathbb{E} \sup _{s \in[0, t]}\left\|u_{n}(s)\right\|^{2 p}\right)^{\frac{1}{2}}\left(\frac{1}{\epsilon} \mathbb{E} \int_{0}^{t}\left\|u_{n}(s)\right\|^{2 p-2}\left(K_{0}^{\prime}\left(1+\left\|u_{n}(s)\right\|^{2}\right)\right) d s\right)^{\frac{1}{2}} \\
& \leq \frac{2 p C}{2} \epsilon \mathbb{E} \sup _{s \in[0, t]}\left\|u_{n}(s)\right\|^{2 p}+\frac{p C K_{0}^{\prime}}{2 \epsilon} \mathbb{E} \int_{0}^{t}\left\|u_{n}(s)\right\|^{2 p-2} d s+\frac{2 p C K_{0}^{\prime}}{2 \epsilon} \int_{0}^{t}\left\|u_{n}(s)\right\|^{2 p} d s \\
& =\frac{2 p C}{2} \epsilon \mathbb{E} \sup _{s \in[0, t]}\left\|u_{n}(s)\right\|^{2 p}+\frac{p C K_{0}^{\prime}}{2 \epsilon} T+\frac{3 p C K_{0}^{\prime}}{2 \epsilon} \mathbb{E} \int_{0}^{t}\left\|u_{n}(s)\right\|^{2 p} d s .
\end{aligned}
$$

By virtue of the Taylor formula, we have

$$
\left|\|x+h\|^{2 p}-\|x\|^{2 p}-2 p\|x\|^{2(p-1)}((x, h))\right| \leq C_{p}\left[\|x\|^{2(p-1)}\|h\|^{2}+\|h\|^{2 p}\right] .
$$


Combining (34) and conditions (d3)-(d4), we find

$$
\begin{aligned}
\mathbb{E} I_{2}(t) & \leq \mathbb{E} \int_{0}^{t} \int_{Z}\left\|u_{n}(s-)+\widetilde{\sigma}_{n}\left(s, u_{n}(s-), z\right)\right\|^{2 p} \\
& -\left\|u_{n}(s-)\right\|^{2 p}-2 p\left\|u_{n}(s-)\right\|^{2(p-1)}\left(\left(u_{n}(s-), \widetilde{\sigma}_{n}\left(s, u_{n}(s-), z\right)\right)\right) N(d s, d z) \\
& \leq C_{p} \mathbb{E} \int_{0}^{t} \int_{Z}\left(\left\|u_{n}(s-)\right\|^{2(p-1)}\left\|\widetilde{\sigma}_{n}\left(s, u_{n}(s-), z\right)\right\|^{2}+\left\|\widetilde{\sigma}_{n}\left(s, u_{n}(s-), z\right)\right\|^{2 p}\right) \mu(d z) d s \\
& \leq\left(C_{p} K_{0}^{\prime} T+C_{p} K^{\prime} T\right)+\left(C_{p} K_{0}^{\prime}+C_{p} K^{\prime}\right) \mathbb{E} \int_{0}^{t}\left\|u_{n}(s)\right\|^{2 p} d s \\
& +C_{p} K_{0}^{\prime} C \mathbb{E} \int_{0}^{t}\left\|u_{n}(s)\right\|^{2(p-1)}\left\|u_{n}(s)\right\|_{D(A)}^{2} d s .
\end{aligned}
$$

Therefore, for $I_{1}(t)+I_{2}(t)$, we have

$$
\begin{aligned}
\mathbb{E}\left(I_{1}(t)+I_{2}(t)\right) & \leq\left(\frac{p C K_{0}^{\prime}}{2 \epsilon} T+C_{p} K_{0}^{\prime} T+C_{p} K^{\prime} T\right)+\left(\frac{3 p C K_{0}^{\prime}}{2 \epsilon}+C_{p} K_{0}^{\prime}+C_{p} K^{\prime}\right) \mathbb{E} \int_{0}^{t}\left\|u_{n}(s)\right\|^{2 p} d s \\
& +\frac{2 p C}{2} \epsilon \mathbb{E} \sup _{s \in[0, t]}\left\|u_{n}(s)\right\|^{2 p}+C_{p} K_{0}^{\prime} C \mathbb{E} \int_{0}^{t}\left\|u_{n}(s)\right\|^{2(p-1)}\left\|u_{n}(s)\right\|_{D(A)}^{2} d s .
\end{aligned}
$$

Now, if we put in Lemma 1 ,

$$
\begin{aligned}
& X(t):=\sup _{s \in[0, t]}\left\|u_{n}(s)\right\|^{2 p}, \\
& Y(t):=\widetilde{\alpha} \int_{0}^{t}\left\|u_{n}(s)\right\|^{2(p-1)}\left\|u_{n}(s)\right\|_{D(A)}^{2} d s, t \in[0, T],
\end{aligned}
$$

and choose $\epsilon$ sufficiently small, we get

$$
\begin{aligned}
\mathbb{E}\left(\sup _{s \in[0, T]}\left\|u_{n}(s)\right\|^{2 p}+\int_{0}^{T}\left\|u_{n}(s)\right\|^{2 p-2}\left\|u_{n}(s)\right\|_{D(A)}^{2} d s\right) & \leq C\left(\mathbb{E}\left(\left\|\Pi_{n} u_{0}\right\|^{2 p}\right)+1\right) \\
& \leq C\left(\mathbb{E}\left(\left\|u_{0}\right\|^{2 p}\right)+1\right) .
\end{aligned}
$$

The constant $C$ depends on $p, K_{0}, K, L_{0}$ but is independent of $n$. This completes the proof of 2).

It remains to prove 3$)$. Taking the square on both sides of (33), we have

$$
\begin{aligned}
& \sup _{s \in[0, t]}\left\|u_{n}(s)\right\|^{4}+\widetilde{\alpha}^{2}\left(\int_{0}^{t}\left\|u_{n}(s)\right\|_{D(A)}^{2} d s\right)^{2} \\
& \leq 6\left(\| u_{0}+C T\right)^{2}+6 T C^{2} \int_{0}^{t} \sup _{r \in[0, s]}\left\|u_{n}(r)\right\|^{4} d s \\
& +24 \sup _{s \in[0, t]}\left(\int_{0}^{s} \int_{Z}\left(\left(u_{n}(s-), \widetilde{\sigma}_{n}\left(s, u_{n}(s-), z\right)\right)\right) \tilde{N}(d s, d z)\right)^{2} \\
& +6 \sup _{s \in[0, t]}\left(\int_{0}^{s} \int_{Z}\left\|\widetilde{\sigma}_{n}\left(s, u_{n}(s-), z\right)\right\|^{2} N(d s, d z)\right)^{2} .
\end{aligned}
$$

We are going to estimate the expectation of the last two terms on the right-hand side of (35). By Burkholder-Davis-Gundy's inequality, condition ( $d 3$ ), Young's inequality, and finally (30) we 
have

$$
\begin{aligned}
& \mathbb{E} \sup _{s \in[0, t]}\left(\int_{0}^{s} \int_{Z}\left(\left(u_{n}(s-), \widetilde{\sigma}_{n}\left(s, u_{n}(s-), z\right)\right)\right) \widetilde{N}(d s, d z)\right)^{2} \\
& \leq C \mathbb{E} \int_{0}^{t} \int_{Z}\left\|u_{n}(s-)\right\|^{2}\left\|\widetilde{\sigma}_{n}\left(s, u_{n}(s-), z\right)\right\|^{2} \mu(d z) d s \\
& \leq C \mathbb{E} \sup _{s \in[0, t]}\left\|u_{n}(s)\right\|^{2} \int_{0}^{t} K_{0}^{\prime}\left(1+\left\|u_{n}(s)\right\|^{2}\right) d s \\
& \leq \frac{C}{2} \mathbb{E} \sup _{s \in[0, t]}\left\|u_{n}(s)\right\|^{4}+\frac{C T}{2} \mathbb{E}\left(\int_{0}^{t}\left(K_{0}^{\prime}\right)^{2}\left(1+\left\|u_{n}(s)\right\|^{2}\right)^{2} d s\right) \\
& \leq \frac{C}{2} \mathbb{E} \sup _{s \in[0, t]}\left\|u_{n}(s)\right\|^{4}+\frac{C T}{2}+\frac{C T}{2}\left(K_{0}^{\prime}\right)^{2} \int_{0}^{t} \mathbb{E} \sup _{r \in[0, s]}\left\|u_{n}(r)\right\|^{4} d s \\
& \leq C\left(\mathbb{E}\left(\left\|u_{0}\right\|^{4}\right)+1\right) .
\end{aligned}
$$

On the other hand, using (7), condition (d4) and (30) we have

$$
\begin{aligned}
& \mathbb{E} \sup _{s \in[0, t]}\left(\int_{0}^{s} \int_{Z}\left\|\widetilde{\sigma}_{n}\left(s, u_{n}(s-), z\right)\right\|^{2} N(d s, d z)\right)^{2} \\
& =\mathbb{E} \sup _{s \in[0, t]}\left(\int_{0}^{s} \int_{Z}\left\|\widetilde{\sigma}_{n}\left(s, u_{n}(s-), z\right)\right\|^{2} \widetilde{N}(d s, d z)+\int_{0}^{s} \int_{Z}\left\|\widetilde{\sigma}_{n}\left(s, u_{n}(s-), z\right)\right\|^{2} \mu(d z) d s\right)^{2} \\
& \leq 2 \mathbb{E} \sup _{s \in[0, t]}\left(\int_{0}^{s} \int_{Z}\left\|\widetilde{\sigma}_{n}\left(s, u_{n}(s-), z\right)\right\|^{2} \widetilde{N}(d s, d z)\right)^{2}+2 \mathbb{E} \sup _{s \in[0, t]}\left(\int_{0}^{s} \int_{Z}\left\|\widetilde{\sigma}_{n}\left(s, u_{n}(s-), z\right)\right\|^{2} \mu(d z) d s\right)^{2} . \\
& \leq 2 \mathbb{E} \int_{0}^{t} \int_{Z}\left\|\widetilde{\sigma}_{n}\left(s, u_{n}(s-), z\right)\right\|^{4} \mu(d z) d s+2\left(\int_{0}^{t} \int_{Z}\left\|\widetilde{\sigma}_{n}\left(s, u_{n}(s-), z\right)\right\|^{2} \mu(d z) d s\right)^{2} \\
& \leq 2 \mathbb{E} \int_{0}^{t} K^{\prime}\left(1+\left\|u_{n}(s)\right\|^{4}\right) d s+2 T \mathbb{E} \int_{0}^{T} K_{0}^{\prime}\left(1+\left\|u_{n}(s)\right\|^{4}\right) d s \\
& \leq C\left(\mathbb{E}\left(\left\|u_{0}\right\|^{4}\right)+1\right) .
\end{aligned}
$$

Taking expectation in (35) and using the estimates (36), (37), we obtain

$$
\mathbb{E}\left(\int_{0}^{T}\left\|u_{n}(s)\right\|_{D(A)}^{2} d s\right)^{2} \leq C\left(\mathbb{E}\left(\left\|u_{0}\right\|^{4}\right)+1\right),
$$

which ends the proof of Proposition 4.

Step 3: Passage to the limit in the finite dimensional equations

Proposition 5. There exists a subsequence of $\left\{u_{n}, n \in \mathbb{N}\right\}$ (still denoted by the same symbol) and random processes

$$
\begin{aligned}
& u \in L^{2}([0, T] \times \Omega, d t \times \mathbb{P} ; D(A)) \cap L^{4}\left(\Omega ; L^{r}([0, T] ; V)\right), \\
& \xi \in L^{4}\left(\Omega, \mathcal{F}_{0}, \mathbb{P} ; V\right), \\
& \mathcal{B} \in L^{2}\left([0, T] \times \Omega, d t \times \mathbb{P} ; D(A)^{\prime}\right), \\
& \Phi \in L^{2}([0, T] \times \Omega, d t \times \mathbb{P} ; V), \\
& \mathcal{G} \in \mathcal{M}_{T}^{2}(\mathcal{P} \otimes \mathcal{Z}, d t \times \mathbb{P} \times \nu ; V),
\end{aligned}
$$


such that

$$
\begin{aligned}
& \text { 1) } u_{n} \rightarrow u \text { weakly in } L^{2}([0, T] \times \Omega, d t \times \mathbb{P} ; D(A)) \\
& \text { and weakly star in ' } L^{4}\left(\Omega ; L^{r}([0, T] ; V)\right) \text { for any } r \in[4, \infty) \text {, } \\
& \text { 2) } u_{n}(0) \rightarrow \xi \text { weakly in } L^{4}\left(\Omega, \mathcal{F}_{0}, \mathbb{P} ; V\right) \text {, } \\
& \text { 3) } \widetilde{B}\left(u_{n}, u_{n}\right) \rightarrow \mathcal{B} \text { weakly in } L^{2}\left([0, T] \times \Omega, d t \times \mathbb{P} ; D(A)^{\prime}\right) \text {, } \\
& \text { 4) } \widetilde{F}\left(t, u_{n}\right) \rightarrow \Phi \text { weakly in } L^{2}([0, T] \times \Omega, d t \times \mathbb{P} ; V) \text {, } \\
& \text { 5) } \widetilde{\sigma}\left(t, u_{n}\right) \rightarrow \mathcal{G} \text { weakly in } \mathcal{M}_{T}^{2}(\mathcal{P} \otimes \mathcal{Z}, d t \times \mathbb{P} \times \nu ; V) \text {. }
\end{aligned}
$$

Moreover $u$ is an $V$-valued càdlàg and $\mathbb{F}$-adapted process and for every $t \in[0, T]$, we have

$$
u(t)+\int_{0}^{t} \widetilde{A} u(s) d s+\int_{0}^{t} \mathcal{B}(s) d s=u_{0}+\int_{0}^{t} \Phi(s) d s+\int_{0}^{t} \int_{Z} \mathcal{G}(s, z) \tilde{N}(d s, d z) .
$$

Proof. From Proposition 4, we have

$$
\sup _{n} \mathbb{E} \int_{0}^{T}\left\|u_{n}(s)\right\|_{D(A)}^{2} d s<\infty \text { and } \sup _{n} \mathbb{E} \sup _{0 \leq s \leq T}\left\|u_{n}(s)\right\|^{4}<\infty .
$$

We can then find a function

$$
u \in L^{2}([0, T] \times \Omega, d t \times \mathbb{P} ; D(A)) \cap L^{4}\left(\Omega ; L^{r}([0, T] ; V)\right),
$$

such that the assertions 1) and 2) hold.

From relation (b2) on $\widetilde{B}$ and Proposition 4, we have

$$
\sup _{n} \mathbb{E} \int_{0}^{T}\left\|\widetilde{B}\left(u_{n}(s), u_{n}(s)\right)\right\|_{D(A)^{\prime}}^{2} d s \leq c_{1} \mathbb{E} \int_{0}^{T}\left\|u_{n}(s)\right\|^{2}\left\|u_{n}(s)\right\|_{D(A)}^{2} d s<\infty .
$$

Therefore, there exists an element $\mathcal{B}$ in $L^{2}\left([0, T] \times \Omega, d t \times \mathbb{P} ; D(A)^{\prime}\right)$ such that $\widetilde{B}\left(u_{n}, u_{n}\right)$ converges weakly to $\mathcal{B}$ in $L^{2}\left([0, T] \times \Omega, d t \times \mathbb{P} ; D(A)^{\prime}\right)$ as $n \rightarrow \infty$.

The existence of $\Phi$ in claim 4) follows from the inequality

$$
\begin{aligned}
& \sup _{n} \mathbb{E} \int_{0}^{T}\left\|\widetilde{F}\left(s, u_{n}(s)\right)\right\|^{2} d s \\
& \leq \sup _{n} \mathbb{E} \int_{0}^{T} L_{0}\left(1+\left\|u_{n}(s)\right\|^{2}\right) d s<\infty .
\end{aligned}
$$

To prove claim 5), we observe that

$$
\begin{aligned}
& \sup _{n} \mathbb{E} \int_{0}^{T} \int_{Z}\left\|\widetilde{\sigma}\left(s, u_{n}(s-), z\right)\right\|^{2} \mu(d z) d s \\
& \leq \sup _{n} \mathbb{E} \int_{0}^{T} K_{0}^{\prime}\left(1+\left\|u_{n}(s)\right\|^{2}\right) d s<\infty .
\end{aligned}
$$

Therefore, there exists an element $\mathcal{G} \in \mathcal{M}_{T}^{2}(\mathcal{P} \otimes \mathcal{Z}, d t \times \mathbb{P} \times \mu ; V)$ such that claim 5) holds. Define a $D(A)^{\prime}$-valued process $\mathrm{X}$ by

$$
X(t):=u_{0}-\int_{0}^{t} \widetilde{A} u(s) d s-\int_{0}^{t} \mathcal{B}(s) d s+\int_{0}^{t} \Phi(s) d s+\int_{0}^{t} \mathcal{G}(s, z) \widetilde{N}(d s, d z), t \in[0, T] .
$$

From the convergence results (38)-(43), it is easy to see that $X$ is a $D(A)^{\prime}$ - valued modification of the $V$-valued process $u \in L^{2}([0, T] \times \Omega ; d t \times \mathbb{P} ; D(A))$ and $X(T)=u(T)$, $\mathbb{P}$-a.s.. It then follows 
from [25] that $u$ is an $V$-valued càdlàg $\left(\mathcal{F}_{t}\right)_{t \in[0, T]}$-adapted process, that is $u \in \mathbb{D}([0, T] ; V)$ and satisfies $\mathbb{P}$-a.s.

$$
u(t)+\int_{0}^{t} \widetilde{A} u(s) d s+\int_{0}^{t} \mathcal{B}(s) d s=u_{0}+\int_{0}^{t} \Phi(s) d s+\int_{0}^{t} \int_{Z} \mathcal{G}(s, z) \tilde{N}(d s, d z),
$$

for all $t \in[0, T]$. This ends the proof of Proposition 5 .

In the fourth step, we will show that

$$
\begin{aligned}
& \mathcal{B}(s)=\widetilde{B}(u(s), u(s)), \Phi(s)=\widetilde{F}(s, u(s)), d t \times \mathbb{P}-\text { a.e.; } \\
& \text { and } \mathcal{G}(s, z)=\widetilde{\sigma}(s, u(s-), z), d t \times \mathbb{P} \times \mu-\text { a.e.. }
\end{aligned}
$$

Step4: To prove that $\mathcal{B}(t)=\widetilde{B}(u(t), u(t)), \Phi(t)=\widetilde{F}(t, u(t))$ and $\mathcal{G}(t, z)=\widetilde{\sigma}(t, u(t-), z)$. We follow the approach in $[10,11]$. For each $n \geq 1$, denote

$$
\widetilde{u}_{n}(t)=\Pi_{n} u(t)=\sum_{k=1}^{n}\left(\left(u(t), v_{k}\right)\right) v_{k}
$$

where $\Pi_{n} \in \mathcal{L}\left(V, V_{n}\right)$ is the orthogonal projection of $V$ onto $H_{n}$. From the properties of $\Pi_{n}$, we have

$$
\begin{aligned}
& \left\|\widetilde{u}_{n}(t)\right\| \leq\|u(t)\|, \\
& \left\|\widetilde{u}_{n}(t)\right\|_{D(A)} \leq \kappa\|u(t)\|_{D(A)}, \\
& \left\|u(t)-\Pi_{n} u(t)\right\| \leq\|u(t)\|,
\end{aligned}
$$

where $\kappa=\left(2 \frac{\|\widetilde{A}\|}{\widetilde{\alpha}}\right)^{\frac{1}{2}}$.

From the property $(a 2)$ of $\widetilde{A}$, we also have

$$
\begin{aligned}
\widetilde{\alpha}\left\|u(t)-\Pi_{n} u(t)\right\|_{D(A)}^{2} & \leq\left\langle\widetilde{A}\left(u(t)-\Pi_{n} u(t)\right), u(t)-\Pi_{n} u(t)\right\rangle_{D(A)^{\prime}} \\
& \leq \sum_{k=n+1}^{\infty} \lambda_{k}\left(\left(u(t), v_{k}\right)\right)^{2} \\
& \leq\langle\widetilde{A} u(t), u(t)\rangle_{D(A)^{\prime}} \\
& \leq C\|u(t)\|_{D(A)}^{2} .
\end{aligned}
$$

Therefore, $d t \times \mathbb{P}$ a.e., $[0, T] \times \Omega$, we have

$$
\lim _{n \rightarrow \infty}\left\|u(t)-\Pi_{n} u(t)\right\|_{D(A)}^{2}=0 .
$$

By the Lebesgue dominated convergence theorem, it follows that

$$
\begin{aligned}
& \lim _{n \rightarrow \infty} \mathbb{E} \int_{0}^{T}\left\|u(t)-\Pi_{n} u(t)\right\|_{D(A)}^{2} d t=0, \\
& \lim _{n \rightarrow \infty} \mathbb{E}\left\|u(t)-\Pi_{n} u(t)\right\|_{D(A)}^{2}=0 .
\end{aligned}
$$

We then have

$$
\widetilde{u}_{n}(t)=\Pi_{n} u \rightarrow \mathrm{u} \text { in } L^{2}([0, T] \times \Omega ; d t \times \mathbb{P} ; D(A)) .
$$

To achieve our goal in Step 4, it suffices to prove that the sequence $\left(u_{n}\right)_{n}$ converges to $u$ up to a stopping time. It is the object of the following lemma.

For any integer $M \geq 1$, consider the sequence of stopping times $\left\{\tau_{M}, M \geq 1\right\}$ defined by

$$
\tau_{M}=\inf \left\{t \in[0, T] ;\|u(t)\|^{2}+\int_{0}^{t}\|u(s)\|_{D(A)}^{2} d s \geq M^{2}\right\} \wedge T
$$

We have $\tau_{M} \rightarrow T$, a.s.. 
Lemma 2. one has

$$
\lim _{n \rightarrow \infty} \mathbb{E} \int_{0}^{\tau_{M}}\left\|u_{n}(s)-u(s)\right\|_{D(A)}^{2} d s=0
$$

Proof. Using the strong convergence results (48), it suffices to prove that

$$
\lim _{n \rightarrow \infty} \mathbb{E} \int_{0}^{\tau_{M}}\left\|\Pi_{n} u(s)-u_{n}(s)\right\|_{D(A)}^{2} d s=0 .
$$

The process $u_{n}-\Pi_{n} u$ satisfies

$$
\begin{aligned}
& d\left(u_{n}-\Pi_{n} u\right)+\left[\widetilde{A}\left(u_{n}-\Pi_{n} u\right)+\Pi_{n} \widetilde{B}\left(u_{n}, u_{n}\right)-\Pi_{n} \mathcal{B}(t)\right] d t=\left(\Pi_{n} \widetilde{F}\left(t, u_{n}(t)\right)-\Pi_{n} \Phi(t)\right) d t \\
& +\int_{Z} \Pi_{n}\left(\widetilde{\sigma}\left(t, u_{n}(t-), z\right)-\mathcal{G}(t, z)\right) \widetilde{N}(d t, d z) .
\end{aligned}
$$

Let $\rho(t)=\exp \left(-n_{1} t-n_{2} \int_{0}^{t}\|u(s)\|_{D(A)}^{2} d s\right), 0 \leq t \leq T$, with $n_{1}$ and $n_{2}$ are positive constants to be fixed later. Applying Itô's formula to the process $\rho(t)\left\|u_{n}(t)-\Pi_{n} u(t)\right\|^{2}$, we obtain

$$
\begin{aligned}
& \rho(t)\left\|u_{n}(t)-\Pi_{n} u(t)\right\|^{2}+2 \int_{0}^{t} \rho(s)\left\langle\widetilde{A}\left(u_{n}(s)-\Pi_{n} u(s)\right), u_{n}(s)-\Pi_{n} u(s)\right\rangle d s \\
& =2 \int_{0}^{t} \rho(s)\left\langle\Pi_{n} \mathcal{B}(s)-\Pi_{n} \widetilde{B}\left(u_{n}(s), u_{n}(s)\right), u_{n}(s)-\Pi_{n} u(s)\right\rangle d s \\
& -n_{1} \int_{0}^{t} \rho(s)\left\|u_{n}(s)-\Pi_{n} u(s)\right\|^{2} d s-n_{2} \int_{0}^{t} \rho(s)\|u(s)\|_{D(A)}^{2}\left\|u_{n}(s)-\Pi_{n} u(s)\right\|^{2} d s \\
& +2 \int_{0}^{t} \rho(s)\left(\left(\left(u_{n}-\Pi_{n} u\right)(s), \Pi_{n}\left(\widetilde{F}\left(s, u_{n}(s)\right)-\Phi(s)\right)\right)\right) d s+ \\
& 2 \int_{0}^{t} \int_{Z} \rho(s)\left(\left(\left(u_{n}-\Pi_{n} u\right)(s-), \Pi_{n}\left(\widetilde{\sigma}\left(s, u_{n}(s-), z\right)-\mathcal{G}(s, z)\right)\right)\right) \widetilde{N}(d s, d z) \\
& +\int_{0}^{t} \int_{Z} \rho(s)\left\|\Pi_{n}\left(\widetilde{\sigma}\left(s, u_{n}(s-), z\right)-\mathcal{G}(s, z)\right)\right\|^{2} N(d s, d z) .
\end{aligned}
$$

Now, observe that from the properties $(b 1),(b 3)$ of $\widetilde{B}$, and relation (45), we have for

$$
\begin{aligned}
& \left\langle\Pi_{n} \mathcal{B}(t)-\Pi_{n} \widetilde{B}\left(u_{n}(t), u_{n}(s)\right) ; u_{n}(t)-\Pi_{n} u(t)\right\rangle \\
& \leq \frac{\widetilde{\alpha}}{2}\left\|u_{n}(t)-\Pi_{n} u(t)\right\|_{D(A)}^{2}+\frac{2 c_{1}^{2} \kappa^{2}}{\widetilde{\alpha}}\|u(t)\|_{D(A)}^{2}\left\|u_{n}(t)-\Pi_{n} u(t)\right\|^{2} \\
& +\left\langle\widetilde{B}\left(\Pi_{n} u(t), \Pi_{n} u(t)\right)-\mathcal{B}(t) ; u_{n}(t)-\Pi_{n} u(t)\right\rangle
\end{aligned}
$$

Also from the property $(c 2)$ of $\widetilde{F}$

$$
\begin{aligned}
& 2\left(\left(\left(u_{n}(t)-\Pi_{n} u(t), \Pi_{n}\left(\widetilde{F}\left(t, u_{n}(t)\right)-\Phi(t)\right)\right)\right)\right. \\
& \leq 2\left(\left(\widetilde{F}\left(t, \Pi_{n} u(t)\right)-\Phi(t), u_{n}(t)-\Pi_{n} u(t)\right)\right)+2 L_{F}^{\frac{1}{2}}\left\|u_{n}(t)-\Pi_{n} u(t)\right\|^{2} .
\end{aligned}
$$

On the other hand,

$$
\begin{aligned}
& \left\|\Pi_{n}\left(\widetilde{\sigma}\left(t, u_{n}(t-), z\right)-\mathcal{G}(t, z)\right)\right\|^{2} \\
& \leq 2\left\|\widetilde{\sigma}\left(t, u_{n}(t-), z\right)-\widetilde{\sigma}\left(t, \Pi_{n} u(t), z\right)\right\|^{2}+2\left\|\widetilde{\sigma}(t, u(t-), z)-\widetilde{\sigma}\left(t, \Pi_{n} u(t), z\right)\right\|^{2}+ \\
& 2\left(\left(\widetilde{\sigma}\left(t, u_{n}(t-), z\right)-\mathcal{G}(t, z) ; \widetilde{\sigma}(t, u(t-), z)-\mathcal{G}(t, z)\right)\right)-\|\widetilde{\sigma}(t, u(t-), z)-\mathcal{G}(t, z)\|^{2},
\end{aligned}
$$


and from the properties of $\widetilde{\sigma}$, we have

$$
\begin{aligned}
& \mathbb{E} \int_{0}^{t} \int_{Z} \rho(s)\left\|\Pi_{n}\left(\widetilde{\sigma}\left(s, u_{n}(s-), z\right)-\mathcal{G}(s, z)\right)\right\|^{2} N(d s, d z) \\
& \leq \mathbb{E} \int_{0}^{t} \int_{Z} \rho(s)\left\|\Pi_{n}\left(\widetilde{\sigma}\left(s, u_{n}(s-), z\right)-\mathcal{G}(s, z)\right)\right\|^{2} d \mu(z) d s \\
& \leq 2 \mathbb{E} \int_{0}^{t} L_{\widetilde{\sigma}} \rho(s)\left\|u_{n}(s-)-\Pi_{n} u(s)\right\|^{2} d s+2 \mathbb{E} \int_{0}^{t} L_{\widetilde{\sigma}} \rho(s)\left\|u(s-)-\Pi_{n} u(s)\right\|^{2} d s \\
& +2 \mathbb{E} \int_{0}^{t} \int_{Z} \rho(s)\left(\left(\widetilde{\sigma}\left(s, u_{n}(s-), z\right)-\mathcal{G}(s, z) ; \widetilde{\sigma}(s, u(s-), z)-\mathcal{G}(s, z)\right)\right) d \mu(z) d s \\
& -\mathbb{E} \int_{0}^{t} \int_{Z} \rho(s)\|\widetilde{\sigma}(s, u(s-), z)-\mathcal{G}(s, z)\|^{2} d \mu(z) d s .
\end{aligned}
$$

Taking the expectation in (51) with the estimates (52)-(55), we arrive at

$$
\begin{aligned}
& \mathbb{E}\left(\rho\left(\tau_{M}\right)\left\|u_{n}\left(\tau_{M}\right)-\Pi_{n} u\left(\tau_{M}\right)\right\|^{2}\right)+\frac{\widetilde{\alpha}}{2} \mathbb{E} \int_{0}^{\tau_{M}} \rho(s)\left\|u_{n}(s)-\Pi_{n} u(s)\right\|_{D(A)}^{2} d s \\
& \leq \frac{2 c_{1}^{2} \kappa^{2}}{\widetilde{\alpha}} \mathbb{E} \int_{0}^{\tau_{M}} \rho(s)\|u(s)\|_{D(A)}^{2}\left\|u_{n}(s)-\Pi_{n} u(s)\right\|^{2} d s \\
& +\mathbb{E} \int_{0}^{\tau_{M}} \rho(s)\left\langle\widetilde{B}\left(\Pi_{n} u(s), \Pi_{n} u(s)\right)-\mathcal{B}(s), u_{n}(s)-\Pi_{n} u(s)\right\rangle d s \\
& -n_{1} \mathbb{E} \int_{0}^{\tau_{M}} \rho(s)\left\|u_{n}(s)-\Pi_{n} u(s)\right\|^{2} d s-n_{2} \mathbb{E} \int_{0}^{\tau_{M}} \rho(s)\|u(s)\|_{D(A)}^{2}\left\|u(s)-\Pi_{n} u(s)\right\|^{2} d s+ \\
& 2 \mathbb{E} \int_{0}^{\tau_{M}} \rho(s)\left(\left(\widetilde{F}\left(s, \Pi_{n} u(s)\right)-\Phi(s) ; u_{n}(s)-\Pi_{n} u(s)\right)\right) d s \\
& +2\left(L_{F}^{\frac{1}{2}}+L_{\widetilde{\sigma}}\right) \mathbb{E} \int_{0}^{\tau_{M}} \rho(s)\left\|u_{n}(s)-\Pi_{n} u(s)\right\|^{2} d s \\
& +2 L_{\widetilde{\sigma}} \mathbb{E} \int_{0}^{T} \rho(s)\left\|u(s)-\Pi_{n} u(s)\right\|^{2} d s \\
& +2 \mathbb{E} \int_{0}^{\tau_{M}} \int_{Z} \rho(s)\left(\left(\widetilde{\sigma}\left(s, u_{n}(s-), z\right)-\mathcal{G}(s, z) ; \widetilde{\sigma}(s, u(s-), z)-\mathcal{G}(s, z)\right)\right) d \mu(z) d s \\
& -\mathbb{E} \int_{0}^{\tau_{M}} \int_{Z} \rho(s)\|\widetilde{\sigma}(s, u(s-), z)-\mathcal{G}(s, z)\|^{2} d \mu(z) d s,
\end{aligned}
$$

where property (a2) of $\widetilde{A}$ has been used. The expectation of the stochastic integral with respect to the compensated Poisson measure vanishes.

Taking $n_{2}=\frac{2 c_{1}^{2} \kappa^{2}}{\widetilde{\alpha}}$ and $n_{1}=2\left(L_{F}^{\frac{1}{2}}+L_{\widetilde{\sigma}}\right)$, we rewrite $(56)$ as follows

$$
\begin{aligned}
& \mathbb{E}\left(\rho\left(\tau_{M}\right)\left\|u_{n}\left(\tau_{M}\right)-\Pi_{n} u\left(\tau_{M}\right)\right\|^{2}\right)+\frac{\widetilde{\alpha}}{2} \mathbb{E} \int_{0}^{\tau_{M}} \rho(s)\left\|u_{n}(s)-\Pi_{n} u(s)\right\|_{D(A)}^{2} d s \\
& +\mathbb{E} \int_{0}^{\tau_{M}} \int_{Z} \rho(s)\|\widetilde{\sigma}(s, u(s-), z)-\mathcal{G}(s, z)\|^{2} d \mu(z) d s \\
& \leq \mathbb{E} \int_{0}^{\tau_{M}} \rho(s)\left\langle\widetilde{B}\left(\Pi_{n} u(s), \Pi_{n} u(s)\right)-\mathcal{B}(s), u_{n}(s)-\Pi_{n} u(s)\right\rangle d s \\
& +2 \mathbb{E} \int_{0}^{\tau_{M}} \rho(s)\left(\left(\widetilde{F}\left(s, \Pi_{n} u(s)\right)-\Phi(s) ; u_{n}(s)-\Pi_{n} u(s)\right)\right) d s \\
& +2 L_{\widetilde{\sigma}} \mathbb{E} \int_{0}^{T} \rho(s)\left\|u(s)-\Pi_{n} u(s)\right\|^{2} d s \\
& +2 \mathbb{E} \int_{0}^{\tau_{M}} \int_{Z} \rho(s)\left(\left(\widetilde{\sigma}\left(s, u_{n}(s), z\right)-\mathcal{G}(s, z) ; \widetilde{\sigma}(s, u(s), z)-\mathcal{G}(s, z)\right)\right) d \mu(z) d s .
\end{aligned}
$$


From (48), we have

$$
\lim _{n \rightarrow \infty} \mathbb{E} \int_{0}^{T} \rho(s)\left\|u(s)-\Pi_{n} u(s)\right\|^{2} d s=0
$$

Also, as

$$
\widetilde{\sigma}\left(t, u_{n}(t), z\right) \rightarrow \mathcal{G}(t, z) \text { in } \mathcal{M}_{T}^{2}(\mathcal{P} \times \mathcal{Z}, d t \times \mathbb{P} \times \mu ; V),
$$

and

$$
I_{\left[0, \tau_{M}\right]} \rho(t)(\widetilde{\sigma}(t, u(t), z)-\mathcal{G}(t, z)) \in \mathcal{M}_{T}^{2}(\mathcal{P} \times \mathcal{Z}, d t \times \mathbb{P} \times \mu ; V),
$$

we then have

$$
\lim _{n \rightarrow \infty} \mathbb{E} \int_{0}^{\tau_{M}} \int_{Z} \rho(s)\left(\left(\widetilde{\sigma}\left(s, u_{n}(s), z\right)-\mathcal{G}(s, z) ; \widetilde{\sigma}(s, u(s), z)-\mathcal{G}(s, z)\right)\right) d \mu(z) d s=0 .
$$

On the other hand

$$
\begin{aligned}
& \mathbb{E} \int_{0}^{\tau_{M}} \rho(s)\left\langle\widetilde{B}\left(\Pi_{n} u(s), \Pi_{n} u(s)\right)-\mathcal{B}(s)+\widetilde{F}\left(s, \Pi_{n} u(s)\right)-\Phi(s) ; u_{n}(s)-\Pi_{n} u(s)\right\rangle d s \\
& =\mathbb{E} \int_{0}^{\tau_{M}} \rho(s)\left\langle\widetilde{B}\left(\Pi_{n} u(s), \Pi_{n} u(s)\right)-\widetilde{B}(u(s), u(s)) ; u_{n}(s)-\Pi_{n} u(s)\right\rangle d s \\
& +\mathbb{E} \int_{0}^{\tau_{M}} \rho(s)\left(\left(\widetilde{F}\left(s, \Pi_{n} u(s)\right)-\widetilde{F}(s, u(s)) ; u_{n}(s)-\Pi_{n} u(s)\right)\right) d s \\
& +\mathbb{E} \int_{0}^{\tau_{M}} \rho(s)\left\langle\widetilde{B}(u(s), u(s))+\widetilde{F}(s, u(s))-\Phi(s)-\mathcal{B}(s) ; u_{n}(s)-\Pi_{n} u(s)\right\rangle d s .
\end{aligned}
$$

From (52) and (48), we have

$$
u_{n}-\Pi_{n} u \rightarrow 0 \text { in } L^{2}([0, T] \times \Omega, d t \times \mathbb{P} ; D(A))
$$

as $n \rightarrow \infty$. Moreover, we have

$$
I_{\left[0, \tau_{M}\right]} \rho(t)(\widetilde{F}(t, u(t))+\widetilde{B}(u(t), u(t))-\Phi(t)-\mathcal{B}(t)) \in L^{2}\left([0, T] \times \Omega, d t \times \mathbb{P} ; D(A)^{\prime}\right),
$$

and

$$
I_{\left[0, \tau_{M}\right]} \rho(t)\left(\widetilde{F}\left(t, \Pi_{n} u(t)\right)-\widetilde{F}(t, u(t))\right) \rightarrow 0 \text { in } L^{2}\left([0, T] \times \Omega, d t \times \mathbb{P} ; D(A)^{\prime}\right) .
$$

Therefore

$$
\begin{aligned}
\lim _{n \rightarrow \infty} \mathbb{E} \int_{0}^{\tau_{M}} \rho(s)\left\langle\widetilde{B}(u(s), u(s))+\widetilde{F}(s, u(s))-\Phi(s)-\mathcal{B}(s) ; u_{n}(s)-\Pi_{n} u(s)\right\rangle d s=0, \\
\lim _{n \rightarrow \infty} \mathbb{E} \int_{0}^{\tau_{M}} \rho(s)\left(\left(\widetilde{F}\left(s, \Pi_{n} u(s)\right)-\widetilde{F}(s, u(s)) ; u_{n}(s)-\Pi_{n} u(s)\right)\right) d s=0 .
\end{aligned}
$$

Also, from relations (b2), (44) and (45), we have

$$
\begin{aligned}
& \left\|I_{\left[0, \tau_{M}\right]} \rho(t)\left(\widetilde{B}\left(\Pi_{n} u(t), \Pi_{n} u(t)\right)-\widetilde{B}(u(t), u(t))\right)\right\|_{D(A)^{\prime}} \\
& \leq \kappa c_{1} I_{\left[0, \tau_{M}\right]} \rho(t)\left\|\Pi_{n} u(t)-u(t)\right\|\|u(t)\|_{D(A)}+\|u(t)\|\left\|\Pi_{n} u(t)-u(t)\right\|_{D(A)},
\end{aligned}
$$

and

$$
\left\|I_{\left[0, \tau_{M}\right]} \rho(t)\left(\widetilde{B}\left(\Pi_{n} u(t), \Pi_{n} u(t)\right)-\widetilde{B}(u(t), u(t))\right)\right\|_{D(A)^{\prime}} \leq(3 \kappa+1)\|u(t)\|_{D(A)} \in L^{2}(0, T ; \mathbb{R}) .
$$

Therefore, by Lebesgue dominated convergence and (61), we obtain

$$
\lim _{n \rightarrow \infty} \mathbb{E} \int_{0}^{\tau_{M}} \rho(s)\left\langle\widetilde{B}\left(\Pi_{n} u(s), \Pi_{n} u(s)\right)-\widetilde{B}(u(s), u(s)) ; u_{n}(s)-\Pi_{n} u(s)\right\rangle d s=0 .
$$


Now, combining (58)-(65) and (57), we obtain

$$
\begin{aligned}
& \lim _{n \rightarrow \infty} \mathbb{E}\left(\rho\left(\tau_{M}\right)\left\|u_{n}\left(\tau_{M}\right)-\Pi_{n} u\left(\tau_{M}\right)\right\|^{2}\right)=0, \\
& \lim _{n \rightarrow \infty} \mathbb{E} \int_{0}^{\tau_{M}} \rho(s)\left\|u_{n}(s)-\Pi_{n} u(s)\right\|_{D(A)}^{2} d s=0, \\
& \mathbb{E} \int_{0}^{\tau_{M}} \int_{Z} \rho(s)\|\widetilde{\sigma}(s, u(s-), z)-\mathcal{G}(s, z)\|^{2} d \mu(z) d s=0 .
\end{aligned}
$$

As the sequence $\left\{\tau_{M} ; M \geq 1\right\}$ is converging to $T$, we obtain from (68) that

$$
\mathcal{G}(s, z)=\widetilde{\sigma}(s, u(s-), z), d t \times \mathbb{P} \times \mu-a . e . .
$$

Also, observe that (48) and (67) imply

$$
u_{n} I_{\left[0, \tau_{M}\right]} \rightarrow u I_{\left[0, \tau_{M}\right]} \text { in } L^{2}([0, T] \times \Omega, d t \times \mathbb{P} ; D(A)),
$$

which ends the proof of Lemma 2.

We are now ready to finish Step 4. Indeed for any $w \in L^{\infty}([0, T] \times \Omega, d t \times \mathbb{P} ; D(A))$, we have

$$
\begin{aligned}
& \mathbb{E} \int_{0}^{\tau_{M}}\left\langle\widetilde{B}(u(s), u(s))-\widetilde{B}\left(u_{n}(s), u_{n}(s)\right), w(s)\right\rangle d s \\
& \leq \kappa C\|w\|_{L^{\infty}([0, T] \times \Omega ; D(A))} \mathbb{E} \int_{0}^{\tau_{M}}\left(\left\|u(s)-u_{n}(s)\right\|\|u(s)\|_{D(A)}+\left\|u_{n}(s)\right\|\left\|u(s)-u_{n}(s)\right\|_{D(A)}\right) d s \\
& \leq \kappa C\|w\|_{L^{\infty}([0, T] \times \Omega ; D(A))}\left(\mathbb{E} \int_{0}^{\tau_{M}}\left\|u(s)-u_{n}(s)\right\|^{2} d s\right)^{\frac{1}{2}}\left(\mathbb{E} \int_{0}^{T}\|u(s)\|_{D(A)}^{2} d s\right)^{\frac{1}{2}} \\
& +\left(\mathbb{E} \int_{0}^{\tau_{M}}\left\|u_{n}(s)\right\|^{2} d s\right)^{\frac{1}{2}}\left(\mathbb{E} \int_{0}^{\tau_{M}}\left\|u(s)-u_{n}(s)\right\|_{D(A)}^{2} d s\right)^{\frac{1}{2}}
\end{aligned}
$$

Therefore, by (69)

$$
\lim _{n \rightarrow \infty} \mathbb{E} \int_{0}^{\tau_{M}}\left\langle\widetilde{B}(u(s), u(s))-\widetilde{B}\left(u_{n}(s), u_{n}(s)\right), w(s)\right\rangle d s=0 .
$$

Taking into account (41), it follows from (71) that

$$
\mathbb{E} \int_{0}^{\tau_{M}}\langle\widetilde{B}(u(s), u(s))-\mathcal{B}(s), w(s)\rangle d s=0
$$

for all $w \in L^{\infty}([0, T] \times \Omega ; D(A))$.

Therefore as $\tau_{M} \rightarrow T$ and $L^{\infty}([0, T] \times \Omega ; D(A))$ is dense in $L^{2}([0, T] \times \Omega ; D(A))$, we obtain from (72) that

$$
\mathcal{B}(t)=\widetilde{B}(u(t), u(t)) \text { as elements of } L^{2}\left([0, T] \times \Omega ; D(A)^{\prime}\right) .
$$

We can also prove that

$$
\Phi(t)=\widetilde{F}(t, u(t)) \text { as elements of } L^{2}([0, T] \times \Omega, d t \times \mathbb{P} ; V) .
$$

Consequently $u$ is a solution of (27).

\section{Step 5: Proof of the estimate (22).}

The proof of the estimate (22) follows from the estimates (30), (31) of Proposition 4 and the weak convergence

$$
\begin{aligned}
& u_{n} \rightarrow u \text { weakly star in } L^{4}\left(\Omega ; L^{\infty}(0, T ; V)\right), \\
& u_{n} \rightarrow u \text { weakly in } L^{4}\left(\Omega ; L^{2}(0, T ; D(A))\right) .
\end{aligned}
$$




\section{Step 6: Existence of the pressure.}

The proof follows the same line as in [11]. But we need to estimate the fourth order moment of the stochastic integral with respect to the compensated Poisson measure. This is achieved by using the result in ([26], Corollary 3.1, Remark 3.6 or [40], Lemma 3.1) concerning the maximal inequality for stochastic integral with respect to the compensated Poisson measure.

By (9) , (10), as $u \in L^{4}\left(\Omega ; L^{2}(0, T ; D(A))\right)$ and is $\mathcal{F}_{t}$-progressively measurable, then

$$
\begin{aligned}
& \mathbb{E}\left(\int_{0}^{t}\left\|(u(s) \cdot \nabla)(u(s)-\alpha \Delta u(s))+(\nabla u)^{*}(s) \cdot \Delta u(s)\right\|_{\left(H^{-1}(D)\right)^{3}} d s\right)^{2} \\
& \leq C \mathbb{E}\left(\int_{0}^{t}|A u(s)|^{2} d s\right)^{2}<\infty,
\end{aligned}
$$

for $t \in[0, T]$. Therefore $(u . \nabla)(u-\alpha \Delta u)+(\nabla u)^{*} . \Delta u \in L^{2}\left(\Omega, \mathcal{F}_{t}, \mathbb{P} ; L^{1}\left(0, t ;\left(H^{-1}(D)\right)^{3}\right)\right)$.

On the other hand

$$
u-\alpha \Delta u \in L^{4}\left(\Omega, \mathcal{F}_{t}, \mathbb{P} ; L^{2}\left(0, t ;\left(L^{2}(D)\right)^{3}\right)\right),
$$

consequently $\partial_{t}(u-\alpha \Delta u) \in L^{4}\left(\Omega, \mathcal{F}_{t}, \mathbb{P} ; H^{-1}\left(0, t ;\left(L^{2}(D)\right)^{3}\right)\right)$ for all $t \in[0, T]$.

Also as $u \in L^{4}(\Omega, \mathcal{F}, \mathbb{P} ; \mathbb{D}([0, T] ; V))$ and is $\mathcal{F}_{t}$-progressively measurable, then it follows that

$$
F(t, u) \in L^{4}\left(\Omega, \mathcal{F}_{t}, \mathbb{P} ; L^{2}\left(0, t ;\left(H^{-1}(D)\right)^{3}\right)\right) .
$$

For the stochastic integral, using the result in ([26], Corollary 3.1, Remark 3.6 or [40], Lemma 3.1 ), we have the estimate

$$
\begin{aligned}
& \mathbb{E} \sup _{0 \leq s \leq t}\left|\int_{0}^{s} \int_{Z} \tilde{\sigma}(s, u(s-), z) \widetilde{N}(d s, d z)\right|^{4} \\
& \leq C \sum_{l=1}^{2}\left(\int_{0}^{t} \int_{Z} \mathbb{E}|\widetilde{\sigma}(s, u(s-), z)|^{2^{l}} \mu(d z) d s\right)^{2^{2-l}} \\
& \leq C\left(\mathbb{E} \int_{0}^{t} K_{0}^{\prime}\left(1+\|u(s)\|^{2}\right) d s\right)^{2}+C \mathbb{E} \int_{0}^{t} K^{\prime}\left(1+\|u(s)\|^{4}\right) d s \\
& \leq C T \mathbb{E} \int_{0}^{t} K_{0}^{\prime 2}\left(1+\|u(s)\|^{4}\right) d s+C \mathbb{E} \int_{0}^{t} K^{\prime}\left(1+\|u(s)\|^{4}\right) d s \\
& <\infty
\end{aligned}
$$

since $u \in L^{4}(\Omega, \mathcal{F}, \mathbb{P} ; \mathbb{D}([0, T] ; V))$. Therefore

$$
\int_{0}^{t} \int_{Z} \widetilde{\sigma}(s, u(s-), z) \widetilde{N}(d s, d z) \in L^{4}\left(\Omega, \mathcal{F}_{t} ; L^{\infty}\left(0, t ;\left(L^{2}(D)\right)^{3}\right)\right)
$$

and

$$
\frac{d}{d t} \int_{0}^{t} \int_{Z} \widetilde{\sigma}(s, u(s-), z) \widetilde{N}(d s, d z) \in L^{4}\left(\Omega, \mathcal{F}_{t}, \mathbb{P} ; W^{-1, \infty}\left(0, t ;\left(L^{2}(D)\right)^{3}\right)\right),
$$

for all $t \in[0, T]$.

Also as $u \in L^{4}\left(\Omega ; L^{2}(0, T ; D(A))\right)$, we have

$$
A u-\alpha \Delta(A u) \in L^{4}\left(\Omega, \mathcal{F}_{t}, \mathbb{P} ; L^{2}\left(0, t ;\left(H^{-2}(D)\right)^{3}\right)\right) .
$$

Arguing as in [36], Remark 4.3, one can prove the existence and uniqueness of the pressure $p$.

\section{Step 7: Uniqueness.}

Let $u_{1}$ and $u_{2}$ be two solutions of problem (1). Let $\bar{u}=u_{1}-u_{2}$. Let $\eta$ to be fixed later and define

$$
\pi(t)=\exp \left(-\eta \int_{0}^{t}\left\|u_{2}(s)\right\|_{D(A)}^{2} d s\right), 0 \leq t \leq T .
$$


Applying Itô's formula to the real process $\pi(t)\|\bar{u}(t)\|^{2}$, we have

$$
\begin{aligned}
& \pi(t)\left\|u_{1}(t)-u_{2}(t)\right\|^{2}+2 \int_{0}^{t} \pi(s)\left\langle\widetilde{A}\left(u_{1}(s)-u_{2}(s)\right), u_{1}(s)-u_{2}(s)\right\rangle d s \\
& =2 \int_{0}^{t} \pi(s)\left\langle\widetilde{B}\left(u_{1}(s), u_{1}(s)\right)-\widetilde{B}\left(u_{2}(s), u_{2}(s)\right), u_{1}(s)-u_{2}(s)\right\rangle d s \\
& +2 \int_{0}^{t} \pi(s)\left\langle\widetilde{F}\left(s, u_{1}(s)\right)-\widetilde{F}\left(s, u_{2}(s)\right), u_{1}(s)-u_{2}(s)\right\rangle d s \\
& +2 \int_{0}^{t} \int_{Z} \pi(s)\left(\left(\left(u_{1}-u_{2}\right)(s-), \widetilde{\sigma}\left(s, u_{1}(s-), z\right)-\widetilde{\sigma}\left(s, u_{2}(s-), z\right)\right)\right) \widetilde{N}(d s, d z) \\
& +\int_{0}^{t} \int_{Z}^{t} \pi(s)\left\|\widetilde{\sigma}\left(s, u_{1}(s-), z\right)-\widetilde{\sigma}\left(s, u_{2}(s-), z\right)\right\|^{2} N(d s, d z) \\
& -\eta \int_{0}^{t} \pi(s)\left\|u_{2}(s)\right\|_{D(A)}^{2}\left\|\left(u_{1}-u_{2}\right)(s)\right\|^{2} d s .
\end{aligned}
$$

Using relation $(a 2)$, conditions $(b 1),(b 3),(c 2)$ and $(75)$, we obtain

$$
\begin{aligned}
& \pi(t)\left\|u_{1}(t)-u_{2}(t)\right\|^{2}+\widetilde{\alpha} \int_{0}^{t} \pi(s)\left\|u_{1}(s)-u_{2}(s)\right\|_{D(A)}^{2} d s \\
& \leq 2 c_{1} \int_{0}^{t} \pi(s)\left\|u_{2}(s)\right\|_{D(A)}\left\|u_{1}(s)-u_{2}(s)\right\|_{D(A)}\left\|u_{1}(s)-u_{2}(s)\right\| d s \\
& +2 L_{\widetilde{F}}^{\frac{1}{2}} \int_{0}^{t} \pi(s)\left\|u_{1}(s)-u_{2}(s)\right\|^{2} d s \\
& 2 \int_{0}^{t} \int_{Z}^{t} \pi(s)\left(\left(\left(u_{1}-u_{2}\right)(s-), \widetilde{\sigma}\left(s, u_{1}(s-), z\right)-\widetilde{\sigma}\left(s, u_{2}(s-), z\right)\right)\right) \widetilde{N}(d s, d z) \\
& +\int_{0}^{t} \int_{Z}^{t} \pi(s)\left\|\widetilde{\sigma}\left(s, u_{1}(s-), z\right)-\widetilde{\sigma}\left(s, u_{2}(s-), z\right)\right\|^{2} N(d s, d z) \\
& -\eta \int_{0}^{t} \pi(s)\left\|u_{2}(s)\right\|_{D(A)}^{2}\left\|\left(u_{1}-u_{2}\right)(s)\right\|^{2} d s .
\end{aligned}
$$

By Young's inequality, we have

$$
\begin{aligned}
& 2 c_{1}\left\|u_{2}(s)\right\|_{D(A)}\left\|u_{1}(s)-u_{2}(s)\right\|_{D(A)}\left\|u_{1}(s)-u_{2}(s)\right\| \\
& \leq \frac{\widetilde{\alpha}}{2}\left\|\left(u_{1}-u_{2}\right)(s)\right\|^{2}+\frac{2 c_{1}^{2}}{\widetilde{\alpha}}\left\|u_{2}(s)\right\|_{D(A)}^{2}\left\|\left(u_{1}-u_{2}\right)(s)\right\|^{2} .
\end{aligned}
$$

Taking the expectation in (76) and taking into account of (77), condition (d2), we arrive at

$$
\begin{aligned}
& \mathbb{E} \pi(t)\left\|u_{1}(t)-u_{2}(t)\right\|^{2}+\frac{\widetilde{\alpha}}{2} \mathbb{E} \int_{0}^{t} \pi(s)\left\|u_{1}(s)-u_{2}(s)\right\|_{D(A)}^{2} d s \\
& \leq \frac{2 c_{1}^{2}}{\widetilde{\alpha}} \mathbb{E} \int_{0}^{t} \pi(s)\left\|u_{2}(s)\right\|_{D(A)}^{2}\left\|\left(u_{1}-u_{2}\right)(s)\right\|^{2} d s \\
& +2 L_{\widetilde{F}}^{\frac{1}{2}} \mathbb{E} \int_{0}^{t} \pi(s)\left\|u_{1}(s)-u_{2}(s)\right\|^{2} d s \\
& +L_{\widetilde{\alpha}} \mathbb{E} \int_{0}^{t} \pi(s)\left\|u_{1}(s)-u_{2}(s)\right\|^{2} d s-\eta \mathbb{E} \int_{0}^{t} \pi(s)\left\|u_{2}(s)\right\|_{D(A)}^{2}\left\|\left(u_{1}-u_{2}\right)(s)\right\|^{2} d s .
\end{aligned}
$$

Choosing $\eta=\frac{2 c_{1}^{2}}{\widetilde{\alpha}}$, we rewrite (78) as follows

$$
\mathbb{E} \pi(t)\left\|u_{1}(t)-u_{2}(t)\right\|^{2} \leq\left(2 L_{\widetilde{F}}^{\frac{1}{2}}+L_{\widetilde{\sigma}}\right) \mathbb{E} \int_{0}^{t} \pi(s)\left\|u_{1}(s)-u_{2}(s)\right\|^{2} d s .
$$


The Gronwall's lemma yields

$$
u_{1}(t)-u_{2}(t)=0, \mathbb{P}-a . s . \text { for all } t \in[0, T] .
$$

This completes the proof of the uniqueness and ends the proof of Theorem 1.

\subsection{Proof of Theorem 2}

The proof of Theorem 2 uses the following result ( see [10]).

Lemma 3. Let $\left\{Q_{n} ; n \geq 1\right\} \subset L^{2}(0, T ; \mathbb{R})$ be a sequence of real processes and let $\left\{\sigma_{M} ; M \geq 1\right\}$ be a sequence of $\mathcal{F}_{t}$-stopping time such that $\sigma_{M} \rightarrow T$ as $M \rightarrow \infty, \sup _{n \geq 1} \mathbb{E}\left|Q_{n}(T)\right|^{2}<\infty$ and $\lim _{M \rightarrow \infty} \mathbb{E}\left|Q_{n}\left(\sigma_{M}\right)\right|=0$, for all $n \geq 1$. Then

$$
\lim _{n \rightarrow \infty} \mathbb{E}\left|Q_{n}(T)\right|=0
$$

Proof. Apply Lemma 3 to

$$
Q_{n}(t)=\int_{0}^{t}\left\|u(s)-u_{n}(s)\right\|_{D(A)}^{2} d s
$$

and $\sigma_{M}=\tau_{M}$. Using the estimates (22), (31), the convergence (69) and the uniqueness of $u$, we conclude that the whole sequence $\left(u_{n}\right)_{n}$ of the Galerkin approximation (28) converges to $u$ strongly in $L^{2}([0, T] \times \Omega, d t \times \mathbb{P} ; D(A))$, that is

$$
\lim _{n \rightarrow \infty} \mathbb{E} \int_{0}^{t}\left\|u_{n}(s)-u(s)\right\|_{D(A)}^{2} d s=0
$$

for all $t \in[0, T]$.

Also apply Lemma 3 to

$$
Q_{n}(t)=\left\|u(t)-u_{n}(t)\right\|^{2} \text { and } \sigma_{M}=\tau_{M} .
$$

Using the estimates (22), (30), the convergence (66) and the uniqueness of $u$, we deduce that the whole sequence $\left(u_{n}\right)_{n}$ of the Galerkin approximation (28) satisfies

$$
\lim _{n \rightarrow \infty} \mathbb{E}\left\|u_{n}(t)-u(t)\right\|^{2}=0
$$

for all $t \in[0, T]$, which ends the proof of Theorem 2 .

\section{Exponential stability of solutions}

In this section, we analyse the stability properties of the stationary solutions to problem (1). For this reason, we suppose that $\widetilde{F}(t, v)=\widetilde{F}(v)$ is independent of $\omega$ and $t$. We associate to (26) the deterministic equation

$$
u(t)+\int_{0}^{t} \widetilde{A} u(s) d s+\int_{0}^{t} \widetilde{B}(u(s), u(s)) d s=u_{0}+\int_{0}^{t} \widetilde{F}(u(s)) d s
$$

$\forall t \in[0, T]$

Definition 3. An element $u^{*} \in D(A)$ is said to be a stationary solution of (79) if

$$
\widetilde{A} u^{*}+\widetilde{B}\left(u^{*}, u^{*}\right)=\widetilde{F}\left(u^{*}\right) .
$$

In [13], we have the following result concerning the existence and uniqueness of the stationary solution of (79). 
Lemma 4. Suppose that

$$
\widetilde{\alpha}>2 c_{2}^{2} L_{\widetilde{F}}^{\frac{1}{2}} .
$$

Then there exists a stationary solution $u^{*} \in D(A)$ of (79). If, moreover

$$
\left(\widetilde{\alpha}-2 c_{2}^{2} L_{\widetilde{F}}^{\frac{1}{2}}\right)^{2}>4 c_{1} c_{2}^{2}\|\widetilde{F}(0)\|
$$

then the stationary solution is unique.

Now, we study the stability of the stationary solutions. In particular, we prove that any solution of problem (1) converges to the stationary solution $u^{*}$ exponentially in the mean square and almost surely exponentially (see the definition in $[14,50]$ ). It is third result of this paper and generalizes the result in ([13], Theorem 3.3) to the case of non-Gaussian Lévy noise.

Theorem 3. Let $u^{*} \in D(A)$ be a stationary solution of (79). We assume that $\widetilde{\sigma}$ satisfies for a.e. in $\Omega \times[0, T]$,

$$
\begin{gathered}
\int_{Z}\left\|\widetilde{\sigma}\left(t, u^{*}, z\right)\right\|^{2} \mu(d z) \leq \gamma(t), \\
\int_{Z}\|\widetilde{\sigma}(t, u, z)-\widetilde{\sigma}(t, v, z)\|^{2} \mu(d z) \leq(\zeta+\delta(t))\|u-v\|^{2},
\end{gathered}
$$

for all $u, v \in V$, where $\zeta>0$ is a constant and $\gamma(t), \delta(t)$ are nonnegative integrable functions such that there exist real numbers $\rho>0, M_{\gamma} \geq 1, M_{\delta} \geq 1$ with

$$
\gamma(t) \leq M_{\gamma} e^{-\rho t}, \quad \delta(t) \leq M_{\delta} e^{-\rho t}, \quad t \geq 0 .
$$

Also suppose that

$$
\widetilde{\alpha}>2 c_{1}\left\|u^{*}\right\|+2 L_{\widetilde{F}}^{\frac{1}{2}} c_{2}^{2}+\zeta c_{2}^{2}
$$

Then,

1) any solution $u(t)$ of problem (1) converges to $u^{*}$ exponentially in the mean square. More precisely, there exists real numbers $\eta \in(0, \rho), M_{0}=M_{0}\left(u_{0}\right)>0$ such that

$$
\mathbb{E}\left\|u(t)-u^{*}\right\|^{2} \leq M_{0} e^{-\eta t}, \forall t>0 .
$$

2) any solution $u(t)$ of problem (1) converges to the stationary solution $u^{*}$ almost surely exponentially.

Proof. 1) Let $u(t)$ be a solution of problem (1). Since $\widetilde{\alpha}>2 c_{1}\left\|u^{*}\right\|+2 L_{\widetilde{F}}^{\frac{1}{2}} c_{2}^{2}+\zeta c_{2}^{2}$, we can take a positive real number $\eta \in(0, \rho)$ such that

$$
\widetilde{\alpha}>\eta c_{2}^{2}+2 c_{1}\left\|u^{*}\right\|+2 L_{\widetilde{F}}^{\frac{1}{2}} c_{2}^{2}+\zeta c_{2}^{2} .
$$

Applying the Itô's formula to the processes $\left\|u(t)-u^{*}\right\|^{2}$ and $e^{\eta t}\left\|u(t)-u^{*}\right\|^{2}$, we have

$$
\begin{aligned}
& \left\|u(t)-u^{*}\right\|^{2}+2 \int_{0}^{t}\left\langle\widetilde{A} u(s), u(s)-u^{*}\right\rangle d s+2 \int_{0}^{t}\left\langle\widetilde{B}(u(s), u(s)), u(s)-u^{*}\right\rangle d s \\
& =\left\|u_{0}-u^{*}\right\|^{2}+2 \int_{0}^{t}\left\langle\widetilde{F}(u(s)), u(s)-u^{*}\right\rangle d s+2 \int_{0}^{t} \int_{Z}\left(\left(u(s-)-u^{*}, \widetilde{\sigma}(s, u(s-), z)\right)\right) \widetilde{N}(d s, d z) \\
& +\int_{0}^{t} \int_{Z}\left[\left\|u(s-)-u^{*}+\widetilde{\sigma}(s, u(s-), z)\right\|^{2}-\left\|u(s-)-u^{*}\right\|^{2}-2\left(\left(u(s-)-u^{*}, \widetilde{\sigma}(s, u(s-), z)\right)\right)\right] N(d s, d z),
\end{aligned}
$$


and

$$
\begin{aligned}
& e^{\eta t}\left\|u(t)-u^{*}\right\|^{2}+2 \int_{0}^{t} e^{\eta s}\left\langle\widetilde{A} u(s), u(s)-u^{*}\right\rangle d s+2 \int_{0}^{t} e^{\eta s}\left\langle\widetilde{B}(u(s), u(s)), u(s)-u^{*}\right\rangle d s \\
& =\left\|u_{0}-u^{*}\right\|^{2}+\int_{0}^{t} \eta e^{\eta s}\left\|u(s)-u^{*}\right\|^{2} d s+2 \int_{0}^{t} e^{\eta s}\left\langle\widetilde{F}(u(s)), u(s)-u^{*}\right\rangle d s \\
& +2 \int_{0}^{t} \int_{Z} e^{\eta s}\left(\left(u(s-)-u^{*}, \widetilde{\sigma}(s, u(s-), z)\right)\right) \widetilde{N}(d s, d z) \\
& +\int_{0}^{t} \int_{Z} e^{\eta s}\left[\left\|u(s-)-u^{*}+\widetilde{\sigma}(s, u(s-), z)\right\|^{2}-\left\|u(s-)-u^{*}\right\|^{2}-2\left(\left(u(s-)-u^{*}, \widetilde{\sigma}(s, u(s-), z)\right)\right)\right] N(d s, d z) .
\end{aligned}
$$

Using the fact that

$$
\|x\|^{2}-\|y\|^{2}+\|x-y\|^{2}=2((x-y, x)), \text { for all } x, y \in V,
$$

we rewrite (85) as follows

$$
\begin{aligned}
& e^{\eta t}\left\|u(t)-u^{*}\right\|^{2}+2 \int_{0}^{t} e^{\eta s}\left\langle\widetilde{A} u(s), u(s)-u^{*}\right\rangle d s+2 \int_{0}^{t} e^{\eta s}\left\langle\widetilde{B}(u(s), u(s)), u(s)-u^{*}\right\rangle d s \\
& =\left\|u_{0}-u^{*}\right\|^{2}+\int_{0}^{t} \eta e^{\eta s}\left\|u(s)-u^{*}\right\|^{2} d s+2 \int_{0}^{t} e^{\eta s}\left\langle\widetilde{F}(u(s)), u(s)-u^{*}\right\rangle d s \\
& +2 \int_{0}^{t} \int_{Z} e^{\eta s}\left(\left(u(s-)-u^{*}, \widetilde{\sigma}(s, u(s-), z)\right)\right) \widetilde{N}(d s, d z)+\int_{0}^{t} \int_{Z} e^{\eta s}\|\widetilde{\sigma}(s, u(s-), z)\|^{2} N(d s, d z) .
\end{aligned}
$$

We also know that $u^{*}$ satisfies

$$
\begin{aligned}
& \int_{0}^{t} e^{\eta s}\left\langle\widetilde{A} u^{*}, u(s)-u^{*}\right\rangle d s+\int_{0}^{t} e^{\eta s}\left\langle\widetilde{B}\left(u^{*}, u^{*}\right), u(s)-u^{*}\right\rangle d s \\
& =\int_{0}^{t} e^{\eta s}\left\langle\widetilde{F}\left(u^{*}\right), u(s)-u^{*}\right\rangle d s .
\end{aligned}
$$

Combining (86) and (87), we obtain

$$
\begin{aligned}
& e^{\eta t}\left\|u(t)-u^{*}\right\|^{2}+2 \int_{0}^{t} e^{\eta s}\left\langle\widetilde{A}\left(u(s)-u^{*}\right), u(s)-u^{*}\right\rangle d s+2 \int_{0}^{t} e^{\eta s}\left\langle\widetilde{B}(u(s), u(s))-\widetilde{B}\left(u^{*}, u^{*}\right), u(s)-u^{*}\right\rangle d s \\
& =\left\|u_{0}-u^{*}\right\|^{2}+\int_{0}^{t} \eta e^{\eta s}\left\|u(s)-u^{*}\right\|^{2} d s+2 \int_{0}^{t} e^{\eta s}\left\langle\widetilde{F}(u(s))-\widetilde{F}\left(u^{*}\right), u(s)-u^{*}\right\rangle d s \\
& +2 \int_{0}^{t} \int_{Z} e^{\eta s}\left(\left(u(s-)-u^{*}, \widetilde{\sigma}(s, u(s-), z)\right)\right) \widetilde{N}(d s, d z)+\int_{0}^{t} \int_{Z} e^{\eta s}\|\widetilde{\sigma}(s, u(s-), z)\|^{2} N(d s, d z) .
\end{aligned}
$$

Remark that using the properties of stochastic integral, we have

$$
\mathbb{E} \int_{0}^{t} \int_{Z} e^{\eta s}\left(\left(u(s-)-u^{*}, \widetilde{\sigma}(s, u(s-), z)\right)\right) \widetilde{N}(d s, d z)=0,
$$

and

$$
\begin{aligned}
& \mathbb{E} \int_{0}^{t} \int_{Z} e^{\eta s}\|\widetilde{\sigma}(s, u(s-), z)\|^{2} N(d s, d z) \\
& \leq \mathbb{E} \int_{0}^{t} \int_{Z} e^{\eta s}\|\widetilde{\sigma}(s, u(s-), z)\|^{2} d \mu(z) d s \\
& \leq \mathbb{E} \int_{0}^{t} e^{\eta s}\left[\gamma(s)+(\zeta+\delta(s))\left\|u(s)-u^{*}\right\|^{2}\right] d s
\end{aligned}
$$


where we have used condition (81).

Also observe that by the properties $(b 1),(b 2)$ of $\widetilde{B}$, we have

$$
\begin{aligned}
& \left\langle\widetilde{B}(u(s), u(s))-\widetilde{B}\left(u^{*}, u^{*}\right), u(s)-u^{*}\right\rangle \\
& =\left\langle\widetilde{B}\left(u^{*}, u(s)-u^{*}\right), u(s)-u^{*}\right\rangle \\
& \leq c_{1}\left\|u^{*}\right\|\left\|u(s)-u^{*}\right\|_{D(A)}^{2} .
\end{aligned}
$$

Now, taking the expectation in (88) and combining (89),(90), (91), (25), relation $(a 2)$ and condition $(c 2)$, we arrive at

$$
\begin{aligned}
& \mathbb{E} e^{\eta t}\left\|u(t)-u^{*}\right\|^{2} \leq \mathbb{E}\left\|u_{0}-u^{*}\right\|^{2} \\
& +\left(\eta c_{2}^{2}-\widetilde{\alpha}+2 c_{1}\left\|u^{*}\right\|+2 L_{\widetilde{F}}^{\frac{1}{2}} c_{2}^{2}+\zeta c_{2}^{2}\right) \int_{0}^{t} e^{\eta s} \mathbb{E}\left\|u(s)-u^{*}\right\|_{D(A)}^{2} d s \\
& +\int_{0}^{t} e^{\eta s}\left(\gamma(s)+\delta(s) \mathbb{E}\left\|u(s)-u^{*}\right\|^{2}\right) d s .
\end{aligned}
$$

Since

$$
\widetilde{\alpha}>\eta c_{2}^{2}+2 c_{1}\left\|u^{*}\right\|+2 L_{\widetilde{F}}^{\frac{1}{2}} c_{2}^{2}+\zeta c_{2}^{2},
$$

we deduce that

$$
\mathbb{E} e^{\eta t}\left\|u(t)-u^{*}\right\|^{2} \leq \mathbb{E}\left\|u_{0}-u^{*}\right\|^{2}+\int_{0}^{t} e^{\eta s}\left(\gamma(s)+\delta(s) \mathbb{E}\left\|u(s)-u^{*}\right\|^{2}\right) d s .
$$

Using Gronwall's lemma, we derive that there exists $M_{0}=M_{0}\left(u_{0}\right)>0$ such that

$$
\mathbb{E}\left\|u(t)-u^{*}\right\|^{2} \leq M_{0} e^{-\eta t}, \forall t>0
$$

which proves (84).

2) Let $N$ be a positive integer. By Itô's formula, we have

$$
\begin{aligned}
& \left\|u(t)-u^{*}\right\|^{2}=\left\|u(N)-u^{*}\right\|^{2}-2 \int_{N}^{t}\left\langle\widetilde{A} u(s), u(s)-u^{*}\right\rangle d s \\
& \left.-2 \int_{N}^{t} \widetilde{B}(u(s), u(s)), u(s)-u^{*}\right\rangle d s+2 \int_{N}^{t}\left\langle\widetilde{F}(u(s)), u(s)-u^{*}\right\rangle d s \\
& +2 \int_{N}^{t} \int_{Z}\left(\left(u(s-)-u^{*}, \widetilde{\sigma}(s, u(s-), z)\right)\right) \widetilde{N}(d s, d z)+\int_{N}^{t} \int_{Z}\|\widetilde{\sigma}(s, u(s-), z)\|^{2} N(d s, d z) .
\end{aligned}
$$

for all $t \geq N$.

By the Burkholder-Davis-Gundy's lemma, we have

$$
\begin{aligned}
& 2 \mathbb{E}\left[\sup _{N \leq t \leq N+1} \int_{N}^{t} \int_{Z}\left(\left(u(s-)-u^{*}, \widetilde{\sigma}(s, u(s-), z)\right)\right) \widetilde{N}(d s, d z)\right] \\
& \leq C \mathbb{E}\left(\int_{N}^{N+1} \int_{Z}\left\|u(s)-u^{*}\right\|^{2}\|\widetilde{\sigma}(s, u(s), z)\|^{2} \mu(d z) d s\right)^{\frac{1}{2}} \\
& \leq \frac{1}{2} \mathbb{E} \sup _{N \leq t \leq N+1}\left\|u(t)-u^{*}\right\|^{2}+\eta_{2} \mathbb{E} \int_{N}^{N+1} \int_{Z}\|\widetilde{\sigma}(s, u(s), z)\|^{2} \mu(d z) d s,
\end{aligned}
$$


where the Young's inequality has been used and $\eta_{2}$ is a positive constant.

Taking the supremum over $[N, N+1]$ and the expectation in (94), we arrive at

$$
\begin{aligned}
& \frac{1}{2} \mathbb{E}\left[\sup _{N \leq t \leq N+1}\left\|u(t)-u^{*}\right\|^{2}\right] \leq \mathbb{E}\left\|u(N)-u^{*}\right\|^{2}-\widetilde{\alpha} \int_{N}^{N+1} \mathbb{E}\left\|u(s)-u^{*}\right\|_{D(A)}^{2} d s \\
& +2 c_{1}\left\|u^{*}\right\| \int_{N}^{N+1} \mathbb{E}\left\|u(s)-u^{*}\right\|_{D(A)}^{2}+2 c_{2}^{2} L_{\widetilde{F}}^{\frac{1}{2}} \int_{N}^{N+1} \mathbb{E}\left\|u(s)-u^{*}\right\|_{D(A)}^{2} d s \\
& +\left(1+\eta_{2}\right) \int_{N}^{N+1}\left(\gamma(s)+(\zeta+\delta(s)) \mathbb{E}\left\|u(s)-u^{*}\right\|^{2}\right) d s \\
& \leq \mathbb{E}\left\|u(N)-u^{*}\right\|^{2}+\left(2 c_{1}\left\|u^{*}\right\|-\widetilde{\alpha}+2 c_{2}^{2} L_{\widetilde{F}}^{\frac{1}{2}}\right) \int_{N}^{N+1} \mathbb{E}\left\|u(s)-u^{*}\right\|_{D(A)}^{2} d s \\
& +\left(1+\eta_{2}\right) \int_{N}^{N+1}\left(\gamma(s)+(\zeta+\delta(s)) \mathbb{E}\left\|u(s)-u^{*}\right\|^{2}\right) d s,
\end{aligned}
$$

where we have used the condition (81), relation $(a 2)$, relations $(b 1),(b 2)$ of $\widetilde{B}$ and condition $(c 2)$ of $\widetilde{F}$.

Now, using (93), it follows that

$$
\begin{aligned}
& \frac{1}{2} \mathbb{E}\left(\sup _{N \leq t \leq N+1}\left\|u(t)-u^{*}\right\|^{2}\right) \\
& \leq \mathbb{E}\left\|u(N)-u^{*}\right\|^{2}+\left(1+\eta_{2}\right) \int_{N}^{N+1}\left(\gamma(s)+(\zeta+\delta(s)) \mathbb{E}\left\|u(s)-u^{*}\right\|^{2}\right) d s .
\end{aligned}
$$

Since $\gamma(t) \leq M_{\gamma} e^{-\rho t}, \delta(t) \leq M_{\delta} e^{-\rho t}, \eta \in(0, \rho), M_{\gamma} \geq 1, M_{\delta} \geq 1$, we deduce from (84), that there exists $M_{1}=M_{1}\left(u_{0}\right) \geq 1$ such that

$$
\mathbb{E}\left(\sup _{N \leq t \leq N+1}\left\|u(t)-u^{*}\right\|^{2}\right) \leq M_{1} e^{-\eta N},
$$

and the proof of 2) follows from the Borel-Cantelli lemma as in [14]. This ends the proof of Theorem 3 .

\section{Acknowledgments}

The work of the first author is supported by the Claude Leon Foundation Postdoctoral Fellowship. The second author is supported by a grant from National Research Foundation of South Africa.

\section{References}

[1] S. Albeverio, Z. Brzeźniak and J. L. Wu, Existence of global solutions and invariant measures for stochastic differential equations driven by Poisson type noise with non-Lipschitz coefficients, J. Math. Anal. Appl. 371 (2010) 309-322.

[2] S. Albeverio, J. Wu and T. Zhang, Parabolic SPDEs driven by Poisson white noise, Stoc. Proc. Appl. 74 (1998) 21-36.

[3] B. Birnir, The Kolmogorov-Obukhov statistical theory of turbulence. J. Nonlinear Sci. 23 (2013) 657-688. 
[4] B. Birnir, The Kolmogorov-Obukhov theory of turbulence. A mathematical theory of turbulence. Springer Briefs in Mathematics. Springer, New York, 2013. x+108 pp.

[5] L. Bo, K. Shi and Y. Wang, Variational solutions of dissipative jump-type stochastic evolution equations, J. Math. Anal. Appl. 373 (2011) 111-126.

[6] Z. Brzeźniak, E. Hausenblas. Martingale solutions for Stochastic Equation of Reaction Diffusion Type driven by Lévy noise or Poisson random measure, arXiv: 1010. 5933.

[7] Z. Brzeźniak and J. Zhu. Stochastic beam equations driven by compensated Poisson random measures, arXiv: 1011. 5377.

[8] Z. Brzeźniak, E. Hausenblas and J. Zhu, 2D stochastic Navier-Stokes equations driven by jump noises, Nonlinear Analysis: Theory, Methods and Applications, 79 (2013) 122-139.

[9] Z. Brzeźniak, W. Lei and J. Zhu, Strong solutions for SPDE with locally monotone coefficients driven by Lévy noise, Nonlinear Analysis: Real World Applications, 17 (2014) 283-310.

[10] H. Breckner, Galerkin approximation and the strong solution of the Navier-Stokes equations, Journal of Applied Mathematics and Stochastic Analysis, 13 (2000) 239-259.

[11] T. Caraballo, J. Real and T. Taniguchi, The existence and uniqueness of solutions to stochastic 3-dimensional Lagrangian averaged Navier-Stokes equations, Proceedings of the Royal Society A, 462 (2006) 459-479.

[12] T. Caraballo, A. M. Márquez-Durán and J. Real, On the Stochastic 3D-Lagrangian averaged Navier-Stokes- $\alpha$ model with finite delay, Stoch. Dyn. 5 (2005) 189-200.

[13] T. Caraballo, A. M. Márquez-Durán and J. Real, The asymptotic behavior of a Stochastic 3D LANS- $\alpha$ model. Appl. Math. Optim. 53 (2006) 141-161.

[14] T. Caraballo, J. A. Langa and T. Taniguchi, The exponential behavior and stabilization of stochastic 2D Navier-Stokes equations, J. Differential Equations, 179 (2002) 714-737.

[15] I. Chueshov, A. Millet, Stochastic 2D Hydrodynamical type systems: well posedness and large deviation, Appl. Math. Optim. 61 (2010) 379-420.

[16] D. Coutand, J. Pierce and S. Shkoller, Global well-posedness of weak solutions for the Lagrangian averaged Navier-Stokes equations on bounded domains, Comm. Pure Appl. Anal. 1 (2002) 35-50.

[17] G. Deugoué and M. Sango, On the stochastic 3D Navier-Stokes- $\alpha$ model of fluids turbulence, Abstract and Applied Analysis, 2009 (2009), Article ID 723236, 27p.

[18] G. Deugoué and M. Sango, Weak solutions to stochastic 3D Navier-Stokes- $\alpha$ model of turbulence: $\alpha$-asymptotic. J. Math. Anal. Appl. 384 (2011) 49-62.

[19] G. Deugoué, P. A. Razafimandimby and M. Sango, On the 3-D stochastic magnetohydrodynamic- $\alpha$ model. Stochastic Process. Appl. 122 (2012) 2211-2248.

[20] G. Deugoué and M. Sango, Convergence for a Splitting-Up Scheme for the 3D Stochastic Navier-Stokes- $\alpha$ model. Stoch. Anal. Appl. 32 (2014) 253-279.

[21] Z. Dong and T. G. Xu, One dimensional stochastic Burgers equation driven by Lévy processes, J. Funct. Anal. 243 (2007) 631-678.

[22] Z. Dong, L. Xu and X. Zhang, Invariant measures of stochastic 2D Navier-Stokes equations driven by $\alpha$-stable processes, Elect. Comm. in Probab. 16 (2011), 678-688. 
[23] B. Dubrulle, Intermittency in fully developed turbulence: in log-Poisson statistics and generalized scale covariance. Phys. Rev. Lett. 73 (1994) 959-962.

[24] C. Foias, D. D. Holm and E. Titi, The three dimensional viscous Camassa- Holm equations, and their relation to the Navier-Stokes equations and turbulence theory. J. Dynam. Differential equations, 14 (2002) 1-35.

[25] I. Gyöngy , N. V. Krylov, On stochastics equations with respect to semimartingales, II. Itö formula in Banach spaces. Stochastics, 6 (1982) 153-173.

[26] E. Hausenblas, Existence, Uniqueness and Regularity of Parabolic SPDEs driven by Poisson random measure, Electron. J. Probab. 10 (2005) 1496-1546.

[27] E. Hausenblas, SPDEs driven by Poisson random measures with non Lipschitz coefficients: existence results, Probab. Theory Related Fields, 137 (2007) 161-200.

[28] E. Hausenblas, P. A. Razafimandimby, M. Sango, Martingale solution to equations for differential type fluids of grade two driven by random force of Lévy type, Potential Anal. 38 (2013) 1291-1331.

[29] D.D. Holm, C. Jeffery, S. Kurien, D. Livescu, M.A. Taylor and B. A. Wingate, The LANS- $\alpha$ model for computing turbulence, Los Alamos Sci. 29 (2005) 152-171.

[30] N. Ikeda, S. Watanabe, Stochastic Differential Equations and Diffusion Processes (NorthHolland and Kodansha, Tokyo, 1981).

[31] G. Kallianpur, J. Xiong, Stochastic differential equations in infinite dimensional spaces, Lectures notes- Monograph Series, vol. 26. Institute of Mathematical Statistics, 1996.

[32] C. Knoche, SPDEs in infinite dimension with Poisson noise, C.R. Math. Acad. Sci. Paris, 339 (2004) 647-652.

[33] A. N. Kolmogorov, Dissipation of energy under locally isotropic turbulence. Dokl. Akad. Nauk SSSR, 32 (1941) 16-18.

[34] A. N. Kolmogorov, The local structure of turbulence in incompressible viscous fluid for very large Reynolds number, Dokl. Akad. Nauk SSSR, 30 (1941) 9-13.

[35] A. N. Kolmogorov, A refinement of previous hypotheses concerning the local structure of turbulence in a viscous incompressible fluid at high Reynolds number, J. Fluid Mech. 13 (1962) 82-85.

[36] J. A. Langa, J. Real and J. Simon, Existence and regularity of the pressure for the stochastic Navier-Stokes equations, Appl. Math. Opt. 48 (2003) 195-210.

[37] W. Liu, M. Röckner. SPDE in Hilbert space with locally monotone coefficients, J. Funct. Anal. 259 (2010) 2902-2922.

[38] V. Mandrekar and B. Rüdiger, Existence and Uniqueness of path wise solutions for stochastic integral equations driven by Lévy noise on separable Banach spaces, Stochastics, 78 (2006) 189-212.

[39] C. Marinelli and M. Röckner, Well-posedness and asymptotic behavior for stochastic reactiondiffusion equations with multiplicative Poisson noise, Electron. J. Probab. 49 (2010) 15281555 .

[40] C. Marinelli, C. Prévôt and M. Röckner, Regular dependence on initial data for stochastic evolution equations with multiplicative Poisson noise, J. Funct. Anal. 258 (2010) 616-649. 
[41] J.E. Marsden and S. Shkoller, Global well-posedness for the LANS- $\alpha$ equations on bounded domains, R. Soc. Lond. Philos. Trans. Ser. A Math. Phys. Eng. Sci. 359 (2001) 1449-1468.

[42] A. M. Obukhov, On the distribution of energy in the spectrum of turbulent flow, Dokl. Akad. Nauk SSSR 32 (1941).

[43] A. M. Obukhov, Some specific features of atmospheric turbulence, J. Fluid Mech. 13 (1962) $77-81$.

[44] S. Peszat and J. Zabczyk, Stochastic Partial differential equations with Lévy noise. An evolution approach, Encyclopedia of Mathematics and its applications, Vol. 113 (Cambridge University Press, Cambridge, 2007).

[45] P. A. Razafimandimby and M. Sango, Strong solution for a stochastic model of two dimensional second grade fluids: Existence, uniqueness and asymptotic behaviour, Nonlinear Analysis:Theory Methods and Applications, 75 (2012) 4251-4270.

[46] M. Röckner and T. Zhang, Stochastic evolution equations of jump type: existence, uniqueness and large deviation principles, Potential Anal. 26 (2007) 255-279.

[47] B. Rüdiger, Stochastic integration with respect to compensated Poisson random measures on separable Banach spaces, Stoch. Stoch. Rep. 76 (2004) 213-242.

[48] Z. S. She and E. Leveque, Universal scaling laws in fully developed turbulence, Phys. Rev. Lett. 72 (1994) 336-339.

[49] Z. S She and E. Waymire, Quantized energy cascade and log-Poisson statistics in fully developed turbulence, Phys. Rev. Lett. 74 (1995) 262-265 .

[50] T. Tachim Medjo, The exponential behavior of the stochastic three-dimensional primitive equations with multiplicative noise, Nonlinear Analysis : Real World Applications, 12 (2011) 799-810.

[51] A. Truman, J. L. Wu, Stochastic Burgers equation with Lévy space-time white noise, Probabilistic methods in fluids, 298-323, World Sci. Publ., River Edge, NJ, 2003.

[52] E. Zeidler, Nonlinear Functional Analysis and its applications II/A: Linear Monotone Operators (Springer- Verlag, New York, 1990).

[53] J. Zhu, "A Study of SPDEs w.r.t Compensated Poisson Random Measures and Related Topics", Ph.D. Thesis. The University of York, 2010.

Gabriel Deugoué, Department of Mathematics and Applied Mathematics, University of Pretoria, Pretoria 0002, South Africa and, Department of Mathematics and Computer Science, University of Dschang, P.O. Box 67, Dschang, Cameroon.

E-mail address: agdeugoue@yahoo.fr

Mamadou Sango, Department of Mathematics and Applied Mathematics, University of Pretoria, Pretoria 0002, South Africa

E-mail address: mamadou.sango@up.ac.za 